
[12] S. Mazur et W. Orlicz, Sur les espaces métriques linéaires, II (to be
published in Studia Mathematica).

[13] W. Orlicz, Beiträge zur Theorie der Orthogonalentricklungen, V, Studi Mathematica 6 (1936), p. 20-38.

[14] W. Orlicz, Sur les opérations linéaires dans l'espace des fonctions bornées, ibidem 10 (1949), p. 60-89.

[15] B. L. van der Wa erden, Ein einfaches Beispiel einer nichtdifferenzierbaren stetigen Funktion, Mathematische Zeitschrift 32 (1950), p. 474-475

[16] A. Zygmund, Trigonometrical Series, Monografie Matematyczne, Warszawa-Lwów 1935.

\section{Linear operations in Saks spaces (I)}

by

W. ORLICZ (Poznań).

This paper deals with metric spaces composed of elements of the unit sphere of a linear normed space, the metric of which is defined (see 1.3) by means of another norm, not necessarily homogeneous. The spaces of this kind may be considered as pseudolinear in a certain sense, and some investigations of Banach spaces can be adapted to the spaces of this kind ${ }^{1}$ ).

1.1. Let $X$ be a linear space. A functional $\|x\|$ defined in $X$ will be called a $B$-norm if it satisfies the following conditions:

(a) $\|x\|=0$ if and only if $x=0$,

(b) $\|x+y\| \leqslant\|x\|+\|y\|$,

(c) $\|\vartheta x\|=|\vartheta|\|x\|$, $\vartheta$ being any real number.

Each functional $\|x\|$ satisfying the above conditions (a), (b) and the following one:

$\left(\mathrm{c}^{\prime}\right)$ if the sequence $\left\{\vartheta_{n}\right\}$ of real numbers tends to $\vartheta$ and $\left\|x_{n}-x\right\| \rightarrow 0$, then $\left\|\vartheta_{n} x_{n}-\vartheta x\right\| \rightarrow 0$

will be said to be a $F$-norm.

Any functional $\|x\|$ satisfying the conditions (b) and (c), or (b) and $\left(\mathrm{c}^{\prime}\right)$, will be termed a $B$ - or $F$-pseudonorm respectively.

A Banach space or a Fréchet space is a linear space $X$ provided with a $B$ - or $F$-norm (i.e. Banach norm or Fréchet norm) respectively and such that the distance

$$
d(x, y)=\|x-y\|
$$

makes $X$ a complete metric space.

1) The results of this paper were presented September 26th 1948 at the VI Polish Mathematical Congress in Warsaw. The second part of the present paper (to appear) will deal with investigation of sequences of operations and with applications of the results of part I. 
If this distance does not define a complete space, $X$ will be said to be an incomplete Banach space or incomplete Fréchet space respectively ${ }^{2}$ ).

1.11. We adopt the following symbols to denote some concrete Banach and Fréchet spaces (addition of elements, multiplication by real numbers, and definition of norm having in each case the usually accepted meaning):

$l^{\alpha}$ - the space of the sequences $\left\{a_{n}\right\}$ for which

$$
\sum_{n=1}^{\infty}\left|a_{n}\right| a<\infty
$$

$m$ - the space of the bounded sequences,

$\boldsymbol{m}_{0}$ - the space of the sequences converging to 0 ,

$L^{\alpha}$ - the space of the functions $x=x(t)$ for which

$$
\int_{0}^{1}|x(t)|^{\alpha} d t<\infty
$$

$M$ - the space of the functions which are measurable and equivalent to bounded functions in a closed interval $\langle a, b\rangle$,

$\boldsymbol{S}$ - the space of the functions measurable in $\langle a, b\rangle$ or, more generally, in a $n$-dimensional interval,

$C$ - the space of the functions continuous in $\langle a, b\rangle$

$C^{*}$ - the space of the functions equivalent to continuous functions in $\langle a, b\rangle$,

$\boldsymbol{V}$ - the space of the functions of bounded variation in $\langle a, b\rangle$,

$V^{*}$ - the space of the functions equivalent to functions of bounded variation in $\langle a, b\rangle$,

$\boldsymbol{V} \boldsymbol{C}$ - the subspace of the space $V$, composed of the continuous functions.

The spaces $\boldsymbol{l}^{a}$ and $\boldsymbol{L}^{\alpha}$ are for $0<\alpha<1$ Fréchet spaces, and are not equivalent to any Banach space; the same property has also the space $\boldsymbol{S}$.

2) S. B a nach in his monography Théorie des opérations linéaires, Monografie Matematyczne, Warsaw 1932, calls these spaces spaces of type $B$ and of type $F$ respectively. Since in the recent literature the spaces of type $B$ are generally called Banach spaces, I adopt for the spaces of type $F$ the term Fréchet spaces, for M. Fréchet was the first to call attention to this class Fréchet spaces, for
of abstract spaces.
We write $x_{n}(t) \stackrel{\text { as }}{\rightarrow} x_{0}(t)$ to denote that the sequence $\left\{x_{n}(t)\right\}$ converges asymptotically to $x_{0}(t)$ in the interval considered.

We denote by supess $x(t)$ the least number $k$ for which the set of the elements $t$ satisfying the inequality $x(t)>k$ is a null-set.

1.2. Let \|\| be a $B$ - or $F$-norm in a linear space $X$.

The sequence $\left\{x_{n}\right\}$ of elements of $X$ will be called bounded with respect to the norm \|\| if $\vartheta_{n} \rightarrow 0$ implies $\vartheta_{n} x_{n} \rightarrow 0$ for each sequence $\left\{\vartheta_{n}\right\}$ of real numbers ${ }^{3}$ ).

If any sequence composed of elements of a set $X_{0} \subset X$ is bounded with respect to the norm \|\| , the set $X_{0}$ itself will be said to be bounded with respect to the norm \|\| .

If there is no risk of mistake, we shall omit the words "with respect to the norm" in the above defined term.

A necessary and sufficient condition for a sequence $\left\{x_{n}\right\}$ of elements of $X$ (for a set $X_{0} \subset X$ ) to be bounded is, given any $\varepsilon>0$, the existence of a $\vartheta_{0}>0$ such that $|\vartheta| \leqslant \vartheta_{0}$ implies $\left\|\vartheta x_{n}\right\|<\varepsilon$ for $n=1,2, \ldots \quad\left(\|\vartheta x\|<\varepsilon\right.$ for every $\left.x \in X_{0}\right)$.

1.21. If a sequence $\left\{x_{n}\right\}$ of elements of $X$ is bounded with respect to the norm \|\| , then $\sup _{(n)}\left\|x_{n}\right\|<\infty$.

The boundedness of the sequence implies the existence of a $\delta>0$ such that $\left\|\vartheta x_{n}\right\| \leqslant 1$ for $n=1,2, \ldots$ and $|\vartheta| \leqslant \delta$; putting $k=\mathrm{E}(1 / \delta)$ and $\vartheta=1-k \delta$ we get $\left\|x_{n}\right\| \leqslant k\left\|\delta x_{n}\right\|+\left\|\vartheta x_{n}\right\| \leqslant k+1$.

The condition $\sup _{(n)}\left\|x_{n}\right\|<\infty$ is in the case of a general $F$-norm only necessary for the boundedness of the sequence. In the case, however, of \|\| being a $B$-norm, it is also sufficient; the set $X_{0}$ is bounded with respect to a $B$-norm \|\| if and only if there exists a $K>0$ such that $x \in X_{0}$ implies $\|x\| \leqslant K$.

1.22. The following property of $F$-norms seems to be useful in the sequel:

Given $\varrho>0$ and $\vartheta_{0}>0$, there exists a $\varrho_{1}$ such that $0<\varrho_{1}<\varrho$, and that $\|x\|<\varrho_{1}$ and $|\vartheta| \leqslant \vartheta_{0}$ imply $\|\vartheta x\| \leqslant \varrho$.

3) This concept of boundedness is due to S. Banach; see S. Mazur and W. Orlicz, Uber Folgen linearer Operationen, Studia Mathematica 4 (1933), p. $152-157$. 
1.3. Let $X$ be a Banach space or an incomplete Banach space (fundamental space) with the norm \|\| , and let \|\|$^{*}$ be another norm defined in $X$. In the set $R$ of elements $x \in X$ satisfying the inequality $\|x\| \leqslant 1$ we define the distance between the elements $x, y \in R$ by the formula

$$
d(x, y)=\|x-y\|^{*} \text {. }
$$

If this metric space is complete, it will be termed a Saks space and denoted by $X_{s}$

It should be pointed out that it is not supposed that the space $X$ with the distance defined as equal to $\|x-y\|$ or to $\|x-y\|^{*}$ is a complete space (this is really the case in the example (VII) which will be considered in 1.4, p. 246).

The following notion of limit defined in the whole of the space $X$ is quite naturally associated with every Saks space: a sequence $\left\{x_{n}\right\}$ of elements of $X$ is said to be $(l)$-convergent to $x_{0}$ if there exists a $K$ such that $\left\|x_{n}\right\| \leqslant K$ for $n=1,2, \ldots$ and $\left\|x_{n}-x_{0}\right\|^{*} \rightarrow 0$ (the space $X_{s}$ being complete, this implies $\left\|x_{0}\right\| \leqslant K$ ).

We shall write $x_{n} \stackrel{l}{\rightarrow} x_{0}$ to. denote that the sequence $\left\{x_{n}\right\}$ is (l)-convergent to $x_{0}$; if $x_{n} \in X_{s}, x_{n} \stackrel{l}{\rightarrow} x_{0}$ means that the sequence $\left\{x_{n}\right\}$ tends to $x_{0}$ in the sense of the metric (1).

1.31. Denote by $K\left(x_{0}, \varrho\right)$ the open sphere with the centre $x_{0}$ and radius $\varrho$ in the space $X_{s}$.

We shall consider Saks spaces satisfying some of the following three conditions:

$\left(\Sigma_{1}\right)$ Given any $x_{0} \in X_{s}$ and $\varrho>0$, there exists a $\delta>0$ such that every element $x \in X_{s}$ for which $d(x, 0)<\delta$ can be roritten in the form $x=x_{1}-x_{2}$, with $x_{1}, x_{2} \in K\left(x_{0}, \varrho\right)$.

$\left(\Sigma_{2}\right)$ If $x_{n} \in X_{s}, x_{n} \stackrel{l}{\rightarrow} 0, \varepsilon_{n}>0$ and $\varepsilon_{n} \rightarrow 0$, then there exists an increasing sequence $\left\{k_{n}\right\}$ of indices and a sequence $\left\{\hat{x}_{k_{n}}\right\}$ such that

(i) $\quad d\left(\hat{x}_{k_{n}}, x_{k_{n}}\right)<\varepsilon_{k_{n}}$ for $n=1,2, \ldots$,

(ii) $\sum_{n=1}^{i} \lambda_{n} \hat{x}_{k_{n}} \in X_{s}$ if $\lambda_{n}=0$ or 1 ,

(iii) for each sequence $\lambda=\left\{\lambda_{i}\right\}$ composed of 0 's and $1^{\prime} s$ there exists an element $\hat{x}_{2} \in X_{s}$ such that

$$
\sum_{n=1}^{i} \lambda_{n} \hat{x}_{k_{n}} \stackrel{l}{\rightarrow} \hat{x}_{\lambda} \text { as } i \rightarrow \infty
$$

$\left(\Sigma_{2}^{\prime}\right)$ Let $\varepsilon(x)$ be a positive real-oalued function such that $\lim _{x \rightarrow 0} \varepsilon(x)=0$, and given any $i$ let $\left\{x_{i n}\right\}_{n=1,2, \ldots}$ be a sequence $(l)$-conDergent to 0 . Then there exists a sequence $\left\{k_{n}\right\}$ of indices and a sequence $\left\{\hat{x}_{k_{n}}\right\}$ of elements satisfying the conditions (ii), (iii) and

$$
d\left(\hat{x}_{k_{n}}, x_{n k_{n}}\right)<\varepsilon\left(\left\|x_{n k_{n}}\right\|^{*}\right) \quad \text { for } n=1,2, \ldots
$$

If in a Banach space (with the norm \|\| ) we put $\|x\|^{*}=\|x\|$, the corresponding space $X_{s}$ obviously satisfies all the conditions $\left(\Sigma_{1}\right),\left(\Sigma_{2}\right)$ and $\left(\Sigma_{2}^{\prime}\right)$. The point of the matter is that in the most important applications we choose in $X$ as \|\|$^{*}$ another norm, which permits us to get a pseudo-banachian space $X_{s}$ having such properties as separability and compactness - contrarily to the unit sphere of the Banach space $X^{4}$ ).

1.32. We shall say that the condition $\left(\Sigma_{1}\right)$ is satisfied at the point $x_{0} \in X_{s}$ if there exists a $\delta>0$ such that

$d(x, 0)=\|x\|^{*}<\delta$ implies $x_{0}+x \in X_{s}$ for every $x \in X_{s}$.

Let the condition $\left(\Sigma_{1}\right)$ be satisfied at any point of a set $X_{0}$ dense in $X_{s}$. Then the space $X_{s}$ satisfies the conditions $\left(\Sigma_{1}\right),\left(\Sigma_{2}\right)$ and $\left(\Sigma_{2}^{\prime}\right)$.

We first prove that the condition $\left(\Sigma_{1}\right)$ is satisfied. Given a sphere $K\left(x_{0}, \varrho\right)$, there exists a $x_{2} \in K\left(x_{0}, \varrho / 2\right) X_{0}$; then there exists, by hypothesis, a $\delta>0$ such that $\|y\|^{*}<\delta$ implies $x_{1}=x_{2}+y \in X_{s}$, and we may suppose that $\delta<\varrho / 2$. Since

$$
y=x_{1}-x_{2}, \quad d\left(x_{0}, x_{2}\right)<\varrho / 2,
$$

and $d\left(x_{0}, x_{1}\right) \leqslant\left\|x_{0}-x_{2}\right\|^{*}+\|y\|^{*}<\varrho$, we see that the space $X_{s}$ satisfies the condition $\left(\Sigma_{1}\right)$.

4) The idea of such class of spaces and of using the condition $\left(\Sigma_{1}\right)$ has been suggested to me by the paper of S. Saks, On some functionals, Transactions of the American Mathematical Society 35 (1932), p. 549-556, and was applied first by A. Alexiewicz in his paper On sequences of operations (I), this volume, p. 1-30. The notion of $(l)$-convergence appears in full generality first in the paper of A. Al exi e w icz, On sequences of operations (II), ibidem, p. 200-236, and in bis and in his pactén Comptes Rendus de la Societe des Sciences had been noted by G. Fichtenexamples of linear spaces with $(l)$-convergence had been noted by G. Fichten-
$\mathrm{holz}$ in his paper Sur les fonctionnelles linéaires continues au sens généralisé, Recueil Mathématique de Moscou 4 (1938), p. 199-214.

Studia Mathematica. T. XI. 
We now prove that the condition $\left(\Sigma_{2}\right)$ is satisfied. Let the sequence $\left\{x_{n}\right\}$ be $(l)$-convergent to 0 , and let $\varepsilon_{n}>0$ tend to 0 . We can easily construct a sequence $\left\{k_{i}\right\}_{i=1,2} \ldots$ of indices, a sequence $\left\{\delta_{i}\right\}_{i=0,1, \ldots}$ of positive numbers and a sequence $\left\{y_{i}\right\}$ of points of $X_{0}$ such that for $i=1,2, \ldots$

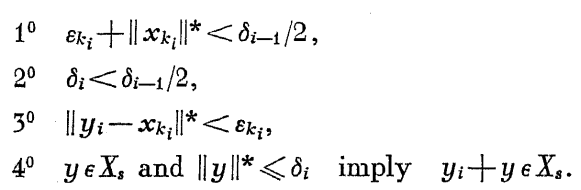

We shall prove that for arbitrary indices $i$ and $l$, and for every sequence $\left\{\lambda_{i}\right\}$ composed of 0 's and 1's

$$
y_{i}+\lambda_{i+1} y_{i+1}+\ldots+\lambda_{i+l} y_{i+l} \in X_{s} \text {. }
$$

Since $1^{0}$ and $3^{0}$ imply $\left\|y_{i+1}\right\|^{*}<\delta_{i} / 2$, by $4^{0}$ we get (2) for $l=1$ and for $i=1,2, \ldots$ Suppose (2) holds for $l=l_{0}-1$ and $i=1,2, \ldots$ Then

$$
\lambda_{i+1} y_{i+1}+\lambda_{i+2} y_{i+2}+\ldots+\lambda_{i+l_{0}} y_{i+l_{0}} \in X_{s}
$$

and by $1^{0}, 2^{0}$ and $3^{0}$

$$
\left\|\lambda_{i+1} y_{i+1}+\ldots+\lambda_{i+l_{0}} y_{i+l_{0}}\right\|^{*} \leqslant \sum_{j=1}^{l_{0}}\left\|y_{i+j}\right\|^{*}<\frac{1}{2} \sum_{j=0}^{l_{0}-1} \delta_{i+j}<\delta_{i} ;
$$

together with $4^{0}$ this implies (2) for $l=l_{0}$ and $i=1,2, \ldots$ Putting $\hat{x}_{k_{i}}=y_{i}$ we obviously get the condition 1.31 (ii); $3^{0}$ implies immediately the condition $1.31(\mathrm{i})$, and, the space $X_{s}$ being complete, the inequality

$$
\sum_{i=1}^{\infty}\left\|y_{i}\right\|^{*}<\frac{1}{2} \sum_{i=0}^{\infty} \delta_{i}<\delta_{0}
$$

implies the condition 1.31(iii).

Finally, we prove the condition $\left(\Sigma_{2}^{\prime}\right)$. Let $\varepsilon(x)$ be a positive real-valued function such that $\lim _{x \rightarrow 0} \varepsilon(x)=0 ;\left\{x_{i n}\right\}_{n=1,2, \ldots}$ being a sequence $(l)$-convergent to 0 , it is easy to see that there exist sequences $\left\{k_{i}\right\},\left\{\delta_{i}\right\}$ and $\left\{y_{i}\right\}$ satisfying the conditions $2^{\circ}$ and $4^{0}$, and such that for $i=1,2, \ldots$.

$$
\varepsilon\left(\left\|x_{i k_{i}}\right\|^{*}\right)+\left\|x_{i k_{i}}\right\|^{*}<\delta_{i-1} / 2,
$$

$3^{\prime 0}$

$$
\left\|y_{i}-x_{i k_{i}}\right\|^{*}<\varepsilon\left(\left\|x_{i k_{i}}\right\|^{*}\right) \text { and } y_{i} \in X_{0} \text {. }
$$

Arguing quite similarly as above we prove that (2) is satisfied for every $i$ and $l$, and putting $\hat{x}_{k_{i}}=y_{i}$ we get a sequence satisfying the conditions of $\left(\Sigma_{2}^{\prime}\right)$.

1.4. We now give some examples of Saks spaces. In each case we indicate the fundamental Banach space $X$, the corresponding norm \|\| and the second norm \|\|$^{*}$. In all these examples, except $\left(I V^{\prime}\right)$ and (VII), the defined Saks spaces are separable; in these.cases we indicate a subset $X_{0}$ enumerable and dense in $X_{s}$.

We omit the proofs of completeness and separability which are easy. The proofs of the conditions $\left(\Sigma_{1}\right)$ and $\left(\Sigma_{2}\right)$ are given in 1.52 .

Examples of Saks spaces satisfying the conditions $\left(\Sigma_{1}\right),\left(\Sigma_{2}\right)$ and $\left(\Sigma_{2}^{\prime}\right)$.

(I) $X$ is the space $\boldsymbol{m}$ of bounded sequences $x=\left\{a_{n}\right\}$ of real numbers, and

$$
\|x\|=\sup _{(n)}\left|a_{n}\right|, \quad\|x\|^{*}=\sum_{n=1}^{\infty} \frac{1}{2^{n}}\left|a_{n}\right| ;
$$

$X_{0}$ is the set of sequences of rationals absolutely less than 1 , and almost all of which are equal to 0 .

(I') $X$ is the space $\boldsymbol{m}_{\varphi}$ of the sequences $x=\left\{a_{n}\right\}$ satisfying the inequality $\left|a_{n}\right|<k \varphi_{n}$, the sequence $\varphi=\left\{\varphi_{n}\right\}$ being a fixed sequence of positive numbers, and

$$
\|x\|=\sup _{(n)}\left|a_{n}\right| / \varphi_{n}, \quad\|x\|^{*}=\sum_{n=1}^{\infty} \frac{1}{2^{n} \varphi_{n}}\left|a_{n}\right| ;
$$

$X_{0}$ is the set of sequences of rationals with the $n$th term absolutely less than $\varphi_{n}$ and almost all terms equal to 0 .

(II) $X$ is the space $\boldsymbol{m}_{T}$ of bounded sequences $x=\left\{a_{n}\right\}$ of real numbers, summable to 0 by a linear sequence-transformation $T$ corresponding to the matrix $\left(a_{i n}\right)$ satisfying the Toeplitz conditions: 


$$
\lim _{i \rightarrow \infty} \alpha_{i n}=0
$$

$$
\text { for }
$$

$\|x\|=\sup _{(n)}\left|a_{n}\right|,\|x\|^{*}=\sup _{(n)}\left|T_{n}(x)\right|+\sum_{n=1}^{\infty} \frac{1}{2^{n}}\left|a_{n}\right|$, where $T_{i}(x)=\sum_{n=1}^{\infty} a_{i n} a_{n} ;$

$X_{0}$ is the same set as in $(\mathrm{I})$.

(III) $X$ is the space $\boldsymbol{M}$ of measurable functions equivalent to bounded functions in $\langle a, b\rangle$, and

$$
\|x\|=\sup _{\langle a, b\rangle} \operatorname{ess}|x(t)|, \quad\|x\|^{*}=\int_{a}^{b}|x(t)| d t ;
$$

$X_{0}$ is the set of all polynomials with rational coefficients absolutely less than 1.

(III') $X$ is the space $M_{\varphi}$ of measurable functions $x=x(t)$ defined in $\langle a, b\rangle$ and satisfying the inequality $|x(t)| \leqslant k \varphi(t)$, where $\varphi(t)=\varphi$ is a fixed measurable positive function, and

$$
\|x\|=\sup _{\langle a, b\rangle} \operatorname{ess}|x(t)| / \varphi(t), \quad\|x\|^{*}=\int_{a}^{b} \frac{|x(t)|}{\varphi(t)} d t ;
$$

$X_{0}$ is the set of functions of the form $m(t) p(t)$, where $m(t)$ is any polynomial with rational coefficients and absolutely less than 1 .

(IV) $X$ is the space $M_{T}$ of measurable functions $x=x(t)$ equivalent to functions bounded in $\langle 0, \infty)$ and such that

$$
\lim _{\tau \rightarrow \infty} T(x, \tau)=0 \text {, }
$$

where

$$
T(x, \tau)=\int_{0}^{\infty} K(\tau, t) x(t) d t
$$

$(a \leqslant \tau<\infty)$

the kernel $K(\tau, t)$ satisfying the conditions:

$$
\int_{0}^{\infty}|K(\tau, t)| d t \leqslant K
$$

for $\tau \geqslant a$,

$$
\lim _{\tau \rightarrow \infty} \int_{0}^{b}|K(\tau, t)| d t=0 \quad \text { for } b \geqslant 0
$$

$$
\|x\|=\sup _{\langle 0, \infty)} \operatorname{ess}|x(t)|, \quad\|x\|^{*}=\max _{\langle a, \infty\rangle}|T(x, \tau)|+\sum_{n=1}^{\infty} \frac{1}{2^{n}} \int_{n-1}^{n}|x(t)| d t ;
$$

$X_{0}$ is the set of functions $x(t)$ for which there exists a rational $t_{0}$ such that

$1^{0}|x(t)| \leqslant 1$ for all $t$,

$2^{0} x(t)$ coincides with a polynomial with rational coefficients for $0 \leqslant t \leqslant t_{0}$,

$3^{0} x(t)=0$ for $t \geqslant t_{0}$.

$\left(\mathrm{IV}^{\prime}\right)\left[\left(\mathrm{IV}^{\prime \prime}\right)\right] X$ is the space $\boldsymbol{M}_{T}^{\prime}\left[\boldsymbol{M}_{T}^{\prime \prime}\right]$ of measurable functions $x=x(t)$ equivalent to bounded measurable functions [to continuous functions] in $\langle 0, \infty)$ and such that there exists the limit (4) with $K(\tau, t)$ satisfying the conditions $(6)-\left(6^{\prime \prime}\right)$;

$$
\|x\|=\sup _{\langle 0, \infty)} \operatorname{ess}|x(t)|, \quad\|x\|^{*}=\max _{\langle a, \infty\rangle}|T(x, \tau)|+\sum_{n=1}^{\infty} \frac{1}{2^{n}}\|x(t)\|_{n},
$$

where $\|x\|_{n}=\sup _{\langle 0, n\rangle}|x(t)| ; X_{0}$ is in the case of the space $M_{T}^{\prime \prime}$ the same set as in the space (IV), but with the restriction that the functions $x(t)$ are continuous.

The space $\boldsymbol{M}_{T}^{\prime}$ is non-separable.

(V) $X$ is the space $D$ of functions $x(t)$ vanishing for $t=a$ and satisfying in $\langle a, b\rangle$ the Lipschitz condition;

$$
\|x\| \sup _{t^{\prime}, t^{\prime \prime} \in\langle a, b\rangle}\left|\frac{x\left(t^{\prime}\right)-x\left(t^{\prime \prime}\right)}{t^{\prime}-t^{\prime \prime}}\right|, \quad\|x\|^{*}=\int_{a}^{b}\left|x^{\prime}(t)\right| d t ;
$$

$X_{0}$ is the set of polynomials with rational coefficients, vanishing for $t=a$, the derivatives of which are absolutely less than 1 .

(VI) $X$ is the space $C^{0}$ of functions $x=x(t)$ bounded in $\langle a, b\rangle$, continuous except at one fixed point $\tau$, and vanishing at $t=\tau$;

$$
\|x\|=\sup _{\langle a, b\rangle}|x(t)|, \quad\|x\|^{*}=\sum_{n=1}^{\infty} \frac{1}{2^{n}}\|x\|_{n} .
$$

To define $\|x\|_{n}$ we choose two sequences $\left\{t_{n}^{\prime}\right\}$ and $\left\{t_{n}^{\prime \prime}\right\}$ such that

$$
\begin{array}{ll}
a<t_{n}^{\prime}<t_{n+1}^{\prime}<\ldots<\tau, & t_{n}^{\prime} \rightarrow \tau, \\
\tau<\ldots<t_{n+1}^{\prime \prime}<t_{n}^{\prime \prime}<b, & t_{n}^{\prime \prime} \rightarrow \tau ;
\end{array}
$$


if $\tau$ is an end-point of $\langle a, b\rangle$, we pick out only one of these sequences. We denote by $\Delta_{n}$ the union of the intervals $\left\langle a, t_{n}^{\prime}\right\rangle$ and $\left\langle t_{n}^{\prime \prime}, b\right\rangle$, or one of these intervals in the case when $\tau$ is an end-point of $\langle a, b\rangle$, and define the pseudonorm $\|x\|_{n}$ by the formula

$$
\|x\|_{n}=\max _{\Delta_{n}}|x(t)|
$$

$X_{0}$ is the set of polynomials of the form $m(t)-m(\tau)$, where $m(t)$ is any polynomial with rational coefficients, such that $|r(t)-r(\tau)|<1$.

Remark. In (VI) we can allow the interval $\langle a, b\rangle$ to be infinite, and $\tau= \pm \infty$; in this case we omit the condition $x(\tau)=0$.

(VII) $X$ is the space $V^{0}$ of bounded functions vanishing at $t=\tau$ and being of bounded variation in all the intervals $\langle a, \tau-\varepsilon\rangle$ and $\langle\tau+\varepsilon, b\rangle$, where the point $\tau$ is fixed between $a$ and $b$, and $\varepsilon$ runs down the set of positive numbers;

$$
x=\sup _{\langle a, b\rangle}|x(t)|, \quad\|x\|^{*}=\sum_{n=1}^{\infty} \frac{1}{2^{n}} \frac{\|x\|_{n}}{1+\|x\|_{n}} .
$$

The pseudonorms $\|x\|_{n}$ are defined as follows: we choose the sequences $\left\{t_{n}^{\prime}\right\}$ and $\left\{t_{n}^{\prime \prime}\right\}$ as in (VI), and put

The space (VII) is non-separable.

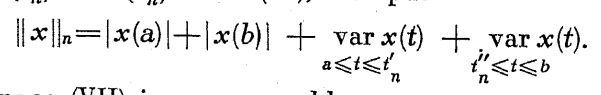

1.51. We now prove two lemmas, which we need in 1.52 .

(A) Suppose that

$$
\begin{array}{ll}
\lim _{n \rightarrow \infty} t_{n}^{(i)}=0 & \text { for } i=1,2, \ldots, \\
\left|t_{n}^{(i)}\right| \leqslant K & \text { for } i, n=1,2, \ldots, \\
\lim _{i \rightarrow \infty} t_{n}^{(i)}=t_{n} & \text { for } n=1,2, \ldots, \\
\lim _{n \rightarrow \infty} t_{n}=0 . &
\end{array}
$$

Then, $\varepsilon$ being any positive number and $s$ being any integer, there exist $a_{s}, a_{s+1}, \ldots, a_{s+r}$ such that

$$
\begin{aligned}
& a_{i} \geqslant 0, \quad \sum_{i=s}^{s+r} \alpha_{i}=1, \\
& \sup _{(n)}\left|\sum_{i=s}^{s+r} \alpha_{i} t_{n}^{(i)}-t_{n}\right|<\varepsilon .
\end{aligned}
$$

To prove this lemma note first that the hypotheses mean that the sequences $t^{(i)}=\left\{t_{n}^{(i)}\right\}_{i=s, s+1, \ldots}$ converge weakly to $\tau_{s}=\left\{t_{n}\right\}$ in the space $\boldsymbol{m}_{0}$, and then apply a general theorem of MAZUR ${ }^{5}$ ).

(B) Let the functions $f_{i}(t)$ be continuous in $\langle a, \infty)$, and suppose that

$$
\begin{aligned}
& \lim _{t \rightarrow \infty} f_{i}(t)=0 \\
& \left|f_{i}(t)\right| \leqslant K
\end{aligned} \quad \text { for } i=1,2, \ldots \text { and } t \in\langle a, \infty),
$$

(iii) the sequence $\left\{f_{i}(t)\right\}$ converges to $f(t)$ uniformly in every finite interval,

$$
\lim _{t \rightarrow \infty} f(t)=0 .
$$

Then, given any $\varepsilon>0$ and an integer $s$, there exist reals $\alpha_{s}, \alpha_{s+1}, \ldots, \alpha_{s+r}$ for which the condition (7) and the condition

$$
\max _{\langle a, \infty\rangle}\left|\sum_{i=s}^{s+r} \alpha_{i} f_{i}(t)-f(t)\right|<\varepsilon
$$

are satisfied.

To prove this lemma put $t_{n}^{(i)}=\max _{\langle a+n-1, a+n\rangle}\left|f_{i}(t)-f(t)\right|$ for $i, n=1,2, \ldots$, and $t_{n}=0$. We easily observe that the hypotheses of lemma (A) are satisfied; hence there exist $\alpha_{s}, \alpha_{s+1}, \ldots, \alpha_{s+r}$ satisfying the conditions ( 7 ) and (8), i. e. such that

$\sup _{\langle n\rangle} \max _{\langle a+n-1, a+n\rangle}\left|\sum_{i=s}^{s+r} \alpha_{i} f_{i}(t)-f(t)\right| \leqslant \sup _{\langle(n)} \sum_{i=s}^{s+r} \alpha_{i} \max _{\langle a+n-1, a+n\rangle}\left|f_{i}(t)-f(t)\right|<\varepsilon$, and this gives $\left(8^{\prime}\right)$.

1.52. We now shall prove that the spaces (I)-(VII) satisfy the conditions $\left(\Sigma_{1}\right),\left(\Sigma_{2}\right)$ and $\left(\Sigma_{2}^{\prime}\right)$. In the sequel $x_{0}$ and $\varrho$ denote respectively the centre and the radius of a sphere $K\left(x_{0}, \varrho\right)$ given in advance.

In the cases of the spaces (I)-(II) and (VI)-(VII) we construct a point $n \in K\left(x_{0}, \varrho\right)$ at which the condition $\left(\Sigma_{1}\right)$ is satisfied; the conclusion results then by 1.32 . The spaces (III)-(V) require a separate proof.

b) S. Mazur, Uber konvexe Mengen in linearen normierlen Räumen, Studia Mathematica 4 (1933), p. 70-80. 
The space (I). Choose $N$ and $\delta$ so that

$$
\sum_{n=N+1}^{\infty} \frac{1}{2^{n}}<\frac{\varrho}{8},
$$

$$
\delta=\frac{1}{2^{N}} \cdot \frac{\varrho}{4},
$$

write $x_{0}=\left\{a_{n}^{(0)}\right\}$, and put

$$
b_{n}=\left\{\begin{array}{cl}
\left(1-\frac{\varrho}{4}\right) a_{n}^{(0)} & \text { for } n=1,2, \ldots, N, \\
0 & \text { elsewhere. }
\end{array}\right.
$$

If $y=\left\{a_{n}\right\} \in X_{s}$ and $d(y, 0)=\|y\|^{*}<\delta$, then writing

$$
z=\left\{a_{n}+b_{n}\right\} \text { and } n=\left\{b_{n}\right\}
$$

we get $\|m\| \leqslant 1$ and $\left|a_{n}\right|<\varrho / 4$ for $n=1,2, \ldots, N$; hence $\|z\| \leqslant 1$ too.

The space $\left(\mathrm{I}^{\prime}\right)$. The proof is analogous as for the space (I).

The space (II). Let $x_{0}=\left\{a_{n}^{(0)}\right\}$; write

$$
\begin{gathered}
a_{n}^{(i)}= \begin{cases}a_{n}^{(0)} & \text { for } n=1,2, \ldots, i, \\
0 & \text { elsewhere, }\end{cases} \\
x_{i}=\left\{a_{n}^{(i)}\right\}, \quad t_{n}=T_{n}\left(x_{0}\right) \quad \text { and } \quad t_{n}^{(i)}=T_{n}\left(x_{i}\right) .
\end{gathered}
$$

The formulae (3) and (3') imply that the sequences $\left\{t_{n}^{(i)}\right\}$ satisfy the hypotheses of Lemma 1.51(A); hence for each integer $s$ there exist $\alpha_{i}$ satisfying the condition (7) and such that

$$
\sup _{(n)}\left|\sum_{i=s}^{s+r} \alpha_{i} T_{n}\left(x_{i}\right)-T_{n}\left(x_{0}\right)\right|<\frac{\varrho}{4} .
$$

Pick out a $s$ such that $N=s$ implies (9), choose $N$ so that $\frac{1}{2^{N}}<\frac{1}{2^{r+s}} \cdot \frac{1}{K}$, define $\delta$ by formula (10), put

$$
\begin{aligned}
& b_{n}=\left(1-\frac{\varrho}{4 K}\right) \sum_{i=s}^{s+r} \alpha_{i} a_{n}^{(i)} \quad \text { for } n=1,2, \ldots, \\
& m=\left\{b_{n}\right\}
\end{aligned}
$$

and let the sequences $z=\left\{a_{n}+b_{n}\right\}$ and $y=\left\{a_{n}\right\}$ have the same meaning as in the proof relative to the space (I). Supposing that $K>1$ we get $\|r\| \leqslant 1$. Since $b_{i}=0$ for $i>s+r$, and since $i \leqslant s+r$ implies

$$
\left|b_{i}\right| \leqslant 1-\frac{\varrho}{4 K} \quad \text { and } \quad\left|a_{i}\right|<\frac{\varrho}{4 K},
$$

we get $\|z\| \leqslant 1$ and

$$
\begin{gathered}
d\left(r, x_{0}\right)=\left\|r-x_{0}\right\|^{*}=\sup _{(n)}\left|T_{n}\left(m-x_{0}\right)\right|+\sum_{n=1}^{\infty} \frac{1}{2^{n}}\left|b_{n}-a_{n}^{(0)}\right| \leqslant \\
\leqslant \sup _{(n)}\left|\sum_{i=s}^{s+r} \alpha_{i} T_{n}\left(x_{i}\right)-T_{n}\left(x_{0}\right)\right|+\frac{\varrho}{4 K} \sup _{(n)}\left|T_{n}\left(\sum_{i=s}^{r+s} a_{i} x_{i}\right)\right|+ \\
+\frac{\varrho}{4 K} \sum_{n=1}^{s} \frac{1}{2^{n}}\left|a_{n}^{(0)}\right|+\sum_{n=s+1}^{\infty} \frac{1}{2^{n}}\left|b_{n}-a_{n}^{(0)}\right|<\frac{\varrho}{4}+\frac{\varrho}{4 K}+\frac{\varrho}{4 K}+\frac{\varrho}{4}<\varrho .
\end{gathered}
$$

The space (III). We first prove the condition $\left(\Sigma_{1}\right)$. We can suppose that $\varrho<4(b-a)$. Let

$$
\delta=\frac{\varrho^{2}}{16(b-a)}, \quad y \in X_{s}, \quad \text { and } \quad d(y, 0)=\|y\|^{*}<\delta .
$$

There exists a set $A \subset\langle a, b\rangle$ such that $|A| \geqslant(b-a)-\frac{\varrho}{4}$ and

$$
\text { teA implies }|y(t)|<\frac{\varrho}{4(b-a)} \text {. }
$$

Put

$$
\begin{aligned}
& m(t)=\left\{\begin{array}{cl}
{\left[1-\frac{\varrho}{4(b-a)}\right] x_{0}(t)} & \text { for } t \in A, \\
0 & \text { for } t \epsilon\langle a, b\rangle-A
\end{array}\right. \\
& z=z(t)=r(t)+y(t) \quad \text { for } t \epsilon\langle a, b\rangle .
\end{aligned}
$$

Obviously $\|m\| \leqslant 1$, and since $|y(t)| \leqslant \frac{\varrho}{4(b-a)}$ for $t \in A$, we get $|z(t)| \leqslant 1$ for $t \in A$; similarly $t e\langle a, b\rangle-A$ implies $|z(t)| \leqslant 1$; hence $\|z\| \leqslant 1$. Finally, writing $y=z-m$, we get 
$d\left(m, x_{0}\right)=\left\|m-x_{0}\right\|^{*}=\frac{\varrho}{4(b-a)_{A}} \int_{A}\left|x_{0}(t)\right| d t+\int_{\langle a, b\rangle-A}\left|x_{0}(t)\right| d t<\frac{\varrho}{4}+\frac{\varrho}{4}=\frac{\varrho}{2}$,

$$
d\left(z, x_{0}\right)=\left\|z-x_{0}\right\|^{*} \leqslant\left\|m-x_{0}\right\|^{*}+\|y\|^{*}<\frac{\varrho}{2}+\delta<\varrho .
$$

We now prove the condition $\left(\Sigma_{2}\right)$. Let $x_{i}=x_{i}(t) \stackrel{l}{\rightarrow} 0, \quad \varepsilon_{i} \rightarrow 0$, and $\varepsilon_{i}>0$. Since

$$
\int_{a}^{b}\left|x_{i}(t)\right| d t \rightarrow 0
$$

$k_{0}$ being chosen freely so that $\varepsilon_{k_{0}}<2(b-a)$, we can easily construct an increasing sequence of indices $\left\{k_{i}\right\}$ such that

$1^{0}$

$$
\varepsilon_{k_{i}}<\varepsilon_{k_{i-1 / 2}}
$$

$2^{0}$

$$
\int_{a}^{b}\left|x_{k_{i}}(t)\right| d t<\frac{\varepsilon_{k_{i-1}}^{2}}{16(b-a)} . \quad \text { for } i=1,2, \ldots
$$

Denote by $A_{i}$ the set of points $t \epsilon\langle a, b\rangle$ satisfying the inequality $\left|x_{k_{i}}(t)\right| \geqslant \frac{\varepsilon_{k_{i-1}}}{4(b-a)}$, and write

$$
B_{i}=A_{i+1}+A_{i+2}+\ldots, \quad C_{i}=\langle a, b\rangle-A_{i} .
$$

Since $2^{0}$ implies $\left|A_{i}\right|<\frac{1}{4} \varepsilon k_{i-1}$, we get by $1^{0}$

$$
\left|B_{i}\right| \leqslant \frac{1}{4} \varepsilon_{k_{i}}+\frac{1}{4} \varepsilon \varepsilon_{i+1}+\ldots<\frac{1}{2} \varepsilon_{k_{i}} .
$$

The function

$$
\hat{x}_{k_{i}}(t)=\left\{\begin{array}{cl}
x_{k_{i}}(t) & \text { for } t \epsilon C_{i}-B_{i}, \\
{\left[1-\frac{\varepsilon_{k_{i}}}{2(b-a)}\right] x_{k_{i}}(t)} & \text { for } t \epsilon A_{i}-B_{i}, \\
0 & \text { for } t \epsilon B_{i}
\end{array}\right.
$$

satisfies the inequality

$$
d\left(\hat{x}_{k_{i}}, x_{k_{i}}\right)=\int_{B_{i}}\left|x_{k_{i}}(t)\right| d t+\frac{\varepsilon_{k_{i}}}{2(b-a)} \int_{A_{i}-B_{i}}\left|x_{k_{i}}(t)\right| d t<\frac{1}{2} \varepsilon_{k_{i}}+\frac{1}{2} \varepsilon k_{i}=\varepsilon_{k_{i}} .
$$

Suppose $t_{\epsilon} A_{n}-\left(A_{n+1}+A_{n+2}+\ldots\right)$; then $t \epsilon B_{1} \cdot B_{2} \cdot \ldots \cdot B_{n-1}$ and

$$
\sum_{i=1}^{\infty}\left|\hat{x}_{k}(t)\right| \leqslant\left[1-\frac{\varepsilon_{k_{n}}}{2(b-a)}\right]+\frac{\varepsilon k_{n}}{4(b-a)}+\frac{\varepsilon_{k_{n+1}}}{4(b-a)}+\ldots \leqslant 1
$$

If $t$ non $\epsilon A_{1}+A_{2}+\ldots$, then $t \in C_{1} \cdot C_{2} \cdot \ldots$; hence

$$
\sum_{i=1}^{\infty}\left|\hat{x}_{k_{i}}(t)\right| \leqslant \frac{\varepsilon_{k_{0}}}{4(b-a)}+\frac{\varepsilon_{k_{1}}}{4(b-a)}+\ldots \leqslant 1 .
$$

If $t$ belongs to an infinity of sets $A_{i}$, i. e. if $t \in \varlimsup_{i \rightarrow \infty} A_{i}$, we have $\left|\varlimsup_{i \rightarrow \infty} A_{i}\right|=0$, and we see that $\lambda_{i}$ being 0 or 1 the element $\sum_{i=1}^{\infty} \lambda_{i} \hat{x}_{k_{i}}=\hat{x}_{\lambda}$ belongs to $X_{s}$; moreover, $\sum_{i=1}^{n} \lambda_{i} \hat{x}_{k_{i}} \rightarrow x_{\lambda}$, for

$$
\left\|\sum_{i=n+1}^{\infty} \lambda_{i} \hat{x}_{k_{i}}\right\|^{*} \leqslant \frac{1}{16(b-a)} \sum_{i=n}^{\infty} \varepsilon_{k_{i}}^{2}
$$

The space (III'). The proofs are similar to those concerning the space (III).

The space (IV). In order to prove the condition $\left(\Sigma_{1}\right)$, we first prove the following lemma:

(*) Given any $b>0$ and $\varepsilon>0$, there exists an $\eta=\eta(b, \varepsilon)>0$ such that

imply

$$
\int_{0}^{b}|x(t)| d t<\eta \quad \text { and } \sup _{t \in\langle 0, b\rangle}|x(t)| \leqslant 1
$$

$$
\max _{\langle a, \infty\rangle}\left|\int_{0}^{b} K(\tau, t) x(t) d t\right|<\varepsilon .
$$

By $\left(6^{\prime}\right)$ there exists a $\Theta$ such that

$$
\tau \geqslant \Theta \text { implies } \int_{0}^{b}|K(\tau, t)| d t<\frac{\varepsilon}{2}
$$

By $\left(6^{\prime \prime}\right)$ the functions $\int_{0}^{b} K(\tau, t) x(t) d t$ are equicontinuous in the interval $a \leqslant \tau \leqslant \Theta$ as $x(t)$ varies over the sphere $|x(t)| \leqslant 1$; hence

$$
\int_{0}^{b} K(\tau, t) x_{n}(t) d t \Rightarrow 0 \text { in }\langle a, \Theta\rangle
$$

as $\int_{0}^{b}\left|x_{n}(t)\right| d t \rightarrow 0$, and $\left|x_{n}(t)\right| \leqslant 1$ in $\langle 0, b\rangle$.

Thus the lemma (*) is proved. 
Now, let $x_{0}=x_{0}(t)$, and

$$
x^{(i)}=x^{(i)}(t)=\left\{\begin{array}{cl}
x_{0}(t) & \text { for } 0 \leqslant t \leqslant i \\
0 & \text { for } t>i .
\end{array}\right.
$$

The conditions $(6)-\left(6^{\prime \prime}\right)$ imply that the functions $T\left(x^{(i)}, \tau\right)$ satisfy the hypotheses of Lemma 1.51(B); hence for every positive integer $s$ there exist $a_{i}$ satisfying the condition (7) and the condition

(11) $\max _{\langle a, \infty)}\left|\sum_{i=s}^{s+r} \alpha_{i} T\left(x^{(i)}, \tau\right)-T\left(x_{0}, \tau\right)\right|<\frac{\varrho}{8}$ with $\frac{\varrho}{8 K}<1$.

Fix $s \geqslant 3$ so that $s=N$ implies the inequality (9); then choose

and put

$$
\eta<\min \left(\eta\left(s+r, \frac{\varrho}{16}\right), \frac{\varrho}{16}, 8 K\right),
$$

$$
0<\delta<\frac{1}{2^{s+r}} \frac{\eta \varrho}{8 K}
$$

If $d(y, 0)=\|y\|^{*}<\delta$, then $\sum_{n=1}^{\infty} \frac{1}{2^{n}} \int_{n-1}^{n}|y(t)| d t<\delta$; hence

$$
\int_{0}^{s+r}|y(t)| d t<\frac{\eta \varrho}{8 K} \text {. }
$$

The set $B$ of those $t$ for which $t \epsilon\langle 0, s+r\rangle$ and $|y(t)| \geqslant \frac{\varrho}{8 K}$ satisfies by (12) the inequality $|B|<\eta$. Let

$$
\begin{aligned}
r_{0}(t)= & \left(1-\frac{\varrho}{8 K}\right) \sum_{i=s}^{s+r} \alpha_{i} x^{(i)}(t) \text { for } t_{\epsilon}\langle 0, \infty), \\
z_{0}(t) & =\left\{\begin{array}{cl}
y(t) & \text { for } t_{\epsilon} B \\
0 & \text { for } \left.t_{\epsilon}<0, \infty\right)-B,
\end{array}\right. \\
D_{0}(t) & =\left\{\begin{array}{cl}
m_{0}(t) & \text { for } t_{\epsilon} B \\
0 & \text { for } \left.t_{\epsilon}<0, \infty\right)-B .
\end{array}\right.
\end{aligned}
$$

By $\left(6^{\prime}\right) \tau \rightarrow \infty$ implies

$$
T\left(m_{0}, \tau\right) \rightarrow 0, \quad T\left(z_{0}, \tau\right) \rightarrow 0 \quad \text { and } \quad T\left(b_{0}, \tau\right) \rightarrow 0 .
$$

It is clear that $\int_{0}^{s+r}\left|z_{0}(t)\right| d t<\eta$; hence by lemma $(*)$

$$
\max _{\langle a, \infty\rangle}\left|T\left(z_{0}, \tau\right)\right|<\frac{\varrho}{16},
$$

and since

$$
\sum_{n=1}^{\infty} \frac{1}{2^{n}} \int_{n-1}^{n}\left|z_{0}(t)\right| d t<\eta<\frac{\varrho}{16},
$$

we get $\left\|z_{0}\right\|^{*}<\varrho / 8$. Similarly $\left\|D_{0}\right\|^{*}<\varrho / 8$.

Put $m=\left(m_{0}-b_{0}\right)-z_{0}$ and $z=\left(m_{0}-b_{0}\right)+\left(y-z_{0}\right)$. The formula

$$
z(t)\left\{\begin{array}{l}
=0 \text { for } t_{\epsilon} B, \\
\leqslant\left|m_{0}(t)\right|+|y(t)| \leqslant\left(1-\frac{\varrho}{8 K}\right)+\frac{\varrho}{8 K}=1 \text { for } t \epsilon\langle 0, \infty)-B
\end{array}\right.
$$

implies $z \in X_{s}$. Similarly $m \in X_{s}$. Obviously $y=z-m$. Now

$$
\begin{gathered}
d\left(m_{0}, x_{0}\right)=\left\|m_{0}-x_{0}\right\|^{*}= \\
=\max _{\langle 0, \infty\rangle}\left|T\left(m_{0}-x_{0}, \tau\right)\right|+\sum_{n=1}^{\infty} \frac{1}{2^{n}} \int_{n-1}^{n}\left|m_{0}(t)-x_{0}(t)\right| d t \leqslant \\
\left.\leqslant \max _{\langle a, \infty\rangle} \sum_{i=s}^{s+r} a_{i} T\left(x^{(i)}, \tau\right)-T\left(x_{0}, \tau\right)\left|+\frac{\varrho}{8 K} \max _{\langle a, \infty\rangle}\right| T \sum_{i=s}^{s+r} a_{i} x^{(i)}, \tau\right) \mid+ \\
+\frac{\varrho}{8 K} \sum_{n=1}^{s} \frac{1}{2^{n}} \int_{n-1}^{n}\left|x_{0}(t)\right| d t+\sum_{n=s+1}^{\infty} \frac{1}{2^{n}} \int_{n-1}^{n}\left|m_{0}(t)-x_{0}(t)\right| d t< \\
<\frac{\varrho}{8}+\frac{\varrho}{8}+\frac{\varrho}{8 K}+\frac{2 \varrho}{8} \leqslant \frac{5 \varrho}{8}, \\
d\left(m, x_{0}\right) \leqslant d\left(m_{0}, x_{0}\right)+\left\|D_{0}\right\|^{*}+\left\|z_{0}\right\|^{*}<\frac{5 \varrho}{8}+\frac{2 \varrho}{8}<\varrho, \\
d\left(z, x_{0}\right) \leqslant d\left(m_{0}, x_{0}\right)+\left\|b_{0}\right\|^{*}+\left\|z_{0}\right\|^{*}+\|y\|^{*}<\frac{5 \varrho}{8}+\frac{2 \varrho}{8}+\eta<\varrho .
\end{gathered}
$$

We now prove the condition $\left(\Sigma_{2}\right)$.

Let $x_{i}=x_{i}(t) \stackrel{l}{\rightarrow} 0, \varepsilon_{i} \rightarrow 0$, and $\varepsilon_{i}>0$. By lemma 1.51 (B) there exist functions $x_{j}^{*}(t)=\sum_{k=s_{j}}^{s_{j}+r_{j}} \alpha_{k} x_{j}^{(k)}(t)$, where

$$
x_{i}^{(k)}(t)=\left\{\begin{array}{cl}
x_{i}(t) & \text { for } 0 \leqslant t \leqslant k \\
0 & \text { for } t>k,
\end{array}\right.
$$

with $s_{j}, r_{j}, \alpha_{k}$ satisfying the conditions of this lemma and such that $d\left(x_{i}^{*}, x_{i}\right)<\varepsilon_{i} / 2$ for $i=1,2, \ldots$ Put $\tau_{j}=s_{j}+r_{j}$ and let $\eta_{i}$ denote 
the number $\eta(b, \varepsilon)$ from lemma (*) corresponding to $b=\tau_{i}$ and $\varepsilon=\varepsilon_{i} / 4$; moreover, let $\eta_{i}<\varepsilon_{i} / 4$ and $\varrho_{i}=\eta_{i} / 2 \tau_{i}$. Choose an increasing sequence of indices $\left\{k_{i}\right\}$ so as to have

$$
\begin{array}{ll}
1^{\prime 0} & \varrho k_{i}<\frac{1}{2} \varrho k_{i-1}, \\
2^{\prime 0} & \int_{0}^{\tau_{i-1}}\left|x_{k_{i}}^{*}(t)\right| d t<\frac{\varrho_{k_{i-1}}^{2}}{4} \text { for } i=1,2, \ldots, \\
3^{\prime 0} & \sum_{i=1}^{\infty}\left\|x_{k_{i}}\right\|^{*}<\infty .
\end{array}
$$

Denoting by $A_{i}$ the set of these $t \epsilon\left\langle 0, \tau_{k_{i-1}}\right)$ for which $\left|x_{k_{i}}^{*}(t)\right| \geqslant \varrho_{k_{i-1} / 2}$, put $B_{i}=A_{i+1}+A_{i+2}+\ldots$ We have $\left|A_{i}\right|<\varrho_{k_{i-1} / 2}$ and $\left|B_{i}\right|<\varrho_{k_{i}}$. Write

$$
\hat{x}_{k_{i}}(t)=\left\{\begin{array}{cl}
\left(1-\varrho_{k_{i}}\right) x_{k_{i}}^{*}(t) & \text { for } t \epsilon\left\langle 0, \tau_{k_{i}}\right\rangle-B_{i} \\
0 & \text { for } t \epsilon B_{i}\left\langle 0, \tau_{k_{i}}\right\rangle \text { and for } t \epsilon\left(\tau_{k_{i}}, \infty\right) .
\end{array}\right.
$$

The following inequality holds:

$$
\int_{0}^{\tau^{k_{k_{i}}}}\left|\hat{x}_{k_{i}}(t)-x_{k_{i}}^{*}(t)\right| d t=\int_{F_{i}}\left|x_{k_{i}}^{*}(t)\right| d t+\varrho_{k_{i}} \int_{G_{i}}\left|x_{k_{i}}^{*}(t)\right| d t<\eta_{k_{i}},
$$

where $F_{i}=\left\langle 0, \tau_{k_{i}}\right\rangle \cdot B_{i}$ and $G_{i}=\left\langle 0, \tau_{k_{i}}\right\rangle-B_{i}$. Hence

$$
\sum_{n=1}^{\infty} \frac{1}{2^{n}} \int_{n-1}^{n}\left|\hat{x}_{k_{i}}(t)-x_{k_{i}}^{*}(t)\right| d t<\eta_{k_{i}}<\frac{1}{4} \varepsilon_{k_{i}}
$$

and by definition of $\eta_{\vec{k}_{i}}$,

$$
\max _{\langle a, \infty)}\left|T\left(\hat{x}_{k_{i}}, \tau\right)-T\left(x_{k_{i}}^{*}, \tau\right)\right|<\frac{1}{4} \varepsilon_{k_{i}},
$$

i.e. $d\left(\hat{x}_{k_{i}}, x_{k_{i}}^{*}\right)<\varepsilon_{k_{i}} / 2$; hence $d\left(\hat{x}_{k_{i}}, x_{k_{i}}\right)<\varepsilon_{k_{i}}$.

Given any $t$, choose $m$ so as to get $t \epsilon\left\langle\tau_{k_{m-1}}, \tau_{k_{m}}\right\rangle$; arguing similarly as in the proof of the condition $\left(\Sigma_{1}\right)$ for the space (III), we can prove that

$$
\sum_{i=1}^{\infty}\left|\hat{x}_{k_{i}}(t)\right|=\sum_{i=m}^{\infty}\left|\hat{x}_{k_{i}}(t)\right| \leqslant 1
$$

except a set of $t$ of measure 0 . Since we have also

$$
\left\|\sum_{i=n}^{m} \lambda_{i} \hat{x}_{k_{i}}\right\|^{*} \leqslant \sum_{i=n}^{m} d\left(\hat{x}_{k_{i}}, x_{k_{i}}\right)+\sum_{i=n}^{m}\left\|x_{k_{i}}\right\|^{*} \leqslant \sum_{i=n}^{m} \varepsilon_{k_{i}}+\sum_{i=n}^{m}\left\|x_{k_{i}}\right\|^{*} \rightarrow 0
$$

as $m, n \rightarrow \infty$, hence $\sum_{i=1}^{n} \lambda_{i} \hat{x}_{i} \stackrel{l}{\rightarrow} x_{\lambda}=\sum_{i=1}^{\infty} \lambda_{i} \hat{x}_{i}$, where $\lambda_{i}$ are 0 's or 1 's arbitrarily choosen.

The proof of the condition $\left(\Sigma_{2}^{\prime}\right)$ is similar.

The space $\left(\mathrm{IV}^{\prime}\right)\left[\left(\mathrm{IV}^{\prime \prime}\right)\right]$. Let $x^{(i)}$ denote the same functions as in the case of the space (IV). Fix $s$ so that $s=N$ implies the inequality (9). There exist $\alpha_{i}$ satisfying (7) and (11). Arguing similarly as above (p. 252-253) we can easily prove that the function $m=m_{0}(t)$ defined by formula (13) satisfies the inequality

$$
d\left(m, x_{0}\right) \leqslant \frac{5}{8} \varrho \text {. }
$$

Choose $\delta>0$ such that

$$
d(0, y)=\|y\|^{*}<\delta \quad \text { implies } \sup _{\langle 0, s+r\rangle} \operatorname{ess}|y(t)|<\frac{\varrho}{8 K}
$$

and put $z=z(t)=m(t)+y(t)$. It follows that $z \in X_{s}$, since

$$
|z(t)| \leqslant\left\{\begin{array}{l}
|m(t)|+|y(t)| \leqslant\left(1-\frac{\varrho}{8 K}\right)+\frac{\varrho}{8 K} \quad \text { for } 0 \leqslant t \leqslant s+r, \\
|y(t)| \leqslant 1 \quad \text { for } t>s+r .
\end{array}\right.
$$

In the case of the space (IV") note that if $x(t)$ is continuous in a finite interval and vanishes elsewhere, we have by $\left(6^{\prime}\right)$ $T(x, \tau) \rightarrow 0$ as $\tau \rightarrow \infty$. Thus we can determine $s$ and $\alpha_{i}$ satisfying the inequality (11). Put

$$
z^{(s+i)}(t)=\left\{\begin{array}{c}
\frac{x^{(s+i)}(s+i)}{s+i-\tau_{i}}[t-(s+i)]+x^{(s+i)}(s+i) \quad \text { for } s+i \leqslant t \leqslant \tau_{i}, \\
0 \quad \text { for } t \in\langle 0, \infty)-\left\langle s+i, \tau_{i}\right\rangle,
\end{array}\right.
$$

where $i=0,1, \ldots, r$, and $\tau_{i}$ is choosen so that

$$
\max _{\langle 0, \infty\rangle}\left|T\left(\mathrm{z}^{(s+i)}, \tau\right)\right|<\frac{\varrho}{4(r+1)} .
$$

This is possible by lemma (*). Put

$$
m(t)=m_{0}(t)+\left(1-\frac{\varrho}{8 K}\right) \sum_{i=s}^{s+r} a_{i} z^{(s+i)}(t) .
$$

It is easy to see that $m(t) \epsilon\left(\mathrm{IV}^{\prime \prime}\right)$ and $d\left(m, x_{0}\right)<\varrho$. Write $p=\mathrm{E}\left(\tau_{r}\right)+1$, and choose $\delta>0$ such that $d(y, 0)=\|y\|^{*}<\delta$ implies $\sup |y(t)|<\varrho / 8 K$. Then $\|m+y\| \leqslant 1$, i. e $w+y \epsilon\left(\mathrm{IV}^{\prime \prime}\right)$. 
The space (V). The formula

$$
x(t)=\int_{a}^{t} y(\tau) d \tau \quad \text { for } t \epsilon\langle a, b\rangle
$$

establishes a one-one mapping between the space $\boldsymbol{D}$ and the space $\boldsymbol{M}$ of measurable functions equivalent to bounded functions. Since

$$
\sup _{t^{\prime}, t^{\prime \prime} \in\langle a, b\rangle}\left|\frac{x\left(t^{\prime}\right)-x\left(t^{\prime \prime}\right)}{t^{\prime}-t^{\prime \prime}}\right|=\sup _{\langle a, b\rangle} \operatorname{ess}|y(t)|, \quad \int_{a}^{b}\left|x^{\prime}(t)\right| d t=\int_{a}^{b}|y(t)| d t,
$$

this mapping is isometrical. The space $\boldsymbol{M}$ satisfies the conditions $\left(\Sigma_{1}\right),\left(\Sigma_{2}\right)$ and $\left(\Sigma_{2}^{\prime}\right)$; hence the space $D$ satisfies these conditions too, for they are invariant under isometrical mappings.

The space (VI). Let e.g. $a<\tau<b$. Let $N$ be a positive integer satisfying the inequality $(9)$, hence such that $1 / 2^{N-1}<\varrho / 2$. Define $\delta$ by formula (10) and write

$$
r(t)=\left\{\begin{array}{cl}
(1-\varrho / 4) x_{0}(t) & \text { for } \quad a \leqslant t \leqslant t_{N-1}^{\prime} \text { and } t_{N-1}^{\prime \prime} \leqslant t \leqslant b, \\
l(t) & \text { for } t_{N-1}^{\prime} \leqslant t \leqslant t_{N}^{\prime} \text { and } t_{N}^{\prime \prime} \leqslant t \leqslant t_{N-1}^{\prime \prime}, \\
0 & \text { for } t_{N}^{\prime} \leqslant t \leqslant t_{N}^{\prime \prime},
\end{array}\right.
$$

where

$$
l(t)= \begin{cases}\frac{m\left(t_{N-1}^{\prime}\right)}{t_{N-1}^{\prime}-t_{N}^{\prime}}\left(t-t_{N-1}^{\prime}\right)+m\left(t_{N-1}^{\prime}\right) & \text { for } t_{N-1}^{\prime} \leqslant t \leqslant t_{N}^{\prime} \\ \frac{m\left(t_{N-1}^{\prime \prime}\right)}{t_{N-1}^{\prime \prime}-t_{N}^{\prime \prime}}\left(t-t_{N-1}^{\prime \prime}\right)+m\left(t_{N-1}^{\prime \prime}\right) & \text { for } t_{N}^{\prime \prime} \leqslant t \leqslant t_{N-1}^{\prime \prime} .\end{cases}
$$

If $d(0, y)=\|y\|^{*}<\delta$, then $\|y\|_{n}<\varrho / 4$ for $n=1,2, \ldots, N$. The function $r(t)$ belongs to $C_{s}^{0}$, and $z(t)=m(t)+y(t) \epsilon C_{s}^{0}$, because $|z(t)|\left\{\begin{array}{l}\leqslant|m(t)|+|y(t)|<\left(1-\frac{\varrho}{4}\right)+\frac{\varrho}{4} \text { for } a \leqslant t \leqslant t_{N}^{\prime} \text { and } t_{N}^{\prime \prime} \leqslant t \leqslant b, \\ =|y(t)| \leqslant 1 \text { for } t_{N}^{\prime} \leqslant t \leqslant t_{N}^{\prime \prime} .\end{array}\right.$ Finally

$$
\begin{gathered}
d\left(m, x_{0}\right)=\left\|m-x_{0}\right\|^{*} \leqslant \\
\leqslant \frac{\varrho}{4} \sum_{n=1}^{N-1} \frac{1}{2^{n}}\left\|x_{0}\right\|_{n}^{*}+\frac{1}{2^{N}}\left(\|m\|_{n}+\left\|x_{0}\right\|_{n}\right)+\sum_{n=N+1}^{\infty} \frac{1}{2^{n}}\left(\|m\|_{n}+\left\|x_{0}\right\|_{n}\right)< \\
<\frac{\varrho}{4}+\frac{\varrho}{2}+2 \frac{\varrho}{8}=\varrho .
\end{gathered}
$$

The space (VII). Choose $\varrho<1$ and $N$ satisfying the inequality (9) and such that $1 / 2^{N}<\varrho / 4$, hence such that $\varrho / 4 K<1$ and $\delta>0$, and that $d(y, 0)=\|y\|^{*}<\delta$ implies $\max \|y\|_{n}<\varrho / 4 K$, where $K=\left\|x_{0}\right\|_{N-1}+1$; we can also suppose $\delta<\varrho / 4$. Define $m(t)$ by the formula differing from that used for the space (VI) by putting $\varrho / 4 K$ instead of $\varrho / 4$, the function $l(t)$ being defined as above by formula (14).

The function $r(t)$ is of bounded variation in $\langle a, b\rangle$, and $\|m\| \leqslant 1$; hence $m \in \boldsymbol{V}_{s}^{0}$. Let $\|y\|^{*}<\delta$ and $z=z(t)=m(t)+y(t)$ Considering that $\sup _{\left\langle a, t_{n}^{\prime}\right\rangle}|x(t)|+\sup _{\left\langle t_{n}^{\prime \prime}, b\right\rangle}|x(t)| \leqslant\|x\|_{n}$, and arguing similarly as for the space $(\mathrm{VI})$, we prove that $\|z\| \leqslant 1$; hence $z \in V_{s}^{0}$. Finally $d\left(r, x_{0}\right)=\left\|m-x_{0}\right\|^{*} \leqslant$

$$
\begin{aligned}
& \leqslant \sum_{n=1}^{N-1} \frac{1}{2^{n}}-\frac{\frac{\varrho}{4 K}\left\|x_{0}\right\|_{n}}{1+\frac{\varrho}{4 K}\left\|x_{0}\right\|_{n}}+\frac{1}{2^{N}} \frac{\left\|m-x_{0}\right\|_{N}}{1+\left\|m-x_{0}\right\|_{N}}+\sum_{n=N+1}^{\infty} \frac{1}{2^{n}} \frac{\left\|m-x_{0}\right\|_{n}}{1+\left\|m-x_{0}\right\|_{n}}< \\
& <\frac{\varrho}{4}+\frac{\varrho}{4}+\frac{\varrho}{8}=\frac{5}{8} \varrho .
\end{aligned}
$$

1.6. We deal in 1.6-1.71 with a class of metric spaces which can be considered as a kind of generalization of Saks spaces. The elements of these spaces are $\mu$-measurable sets or characteristic functions of these sets.

We first define the $\mu$-measurability. Let $T$ be any abstract set. A class $\mathbb{E}$ of subsets of $T$ is called a field, if the union and the difference of two sets of $\mathfrak{E}$ belong to $\mathfrak{E}$. This condition implies that the common part of two sets of $\mathbb{E}$ belongs to $\mathbb{E}$.

Now let $\mathscr{E}$ be a field and suppose a set-function $\mu(E)$, called $\mu$-measure, to be defined for every set $E_{\epsilon} \mathbb{E}$, assuming as values elements of a Banach or Fréchet space. Suppose further the following conditions satisfied:

(a) $E_{1} \cdot E_{2}=0$ implies $\mu\left(E_{1}+E_{2}\right)=\mu\left(E_{1}\right)+\mu\left(E_{2}\right)$,

(b) $E_{1} \subset E_{2}$ implies $\left\|\mu\left(E_{1}\right)\right\| \leqslant\left\|\mu\left(E_{2}\right)\right\|$,

(c) if $\sum_{n=1}^{\infty}\left\|\mu\left(E_{n}\right)\right\|<\infty$ and if the sets $E_{n}$ are mutually disjoint, then the set $\sum_{n=1}^{\infty} E_{n}$ belongs to $\mathbb{E}$, and $\left\|\mu\left(E_{1}+E_{2}+\ldots\right)\right\| \leqslant \sum_{n=1}^{\infty}\left\|\mu\left(E_{n}\right)\right\|$. 
Under these conditions the sets of the field $\mathbb{E}$ will be called measurable. If $\mu(E)=0$, the set $E$ will be said to be a null set. Two sets $E_{1}$ and $E_{2}$ of $E$ which differ by null sets will be said to be $\mu$-equioalent. Two sets $E_{1}$ and $E_{2}$ of $F$, the common part of which is a null set, will be said to be $\mu$-disjoint; in this case we shall write $E_{1} \cdot E_{2}=0$.

Consider the set $X(\mathbb{F})$ the elements of which are the classes of $\mu$-equivalent sets of $\mathfrak{E}$.

We shall prove that $X(\mathbb{E})$ is a complete metric space $\left.{ }^{6}\right)$ if the distance of elements $A$ and $B$ of $X(\widetilde{E})$ is defined by the formula

$$
d(A, B)=\|\mu(A-B)+\mu(B-A)\| .
$$

1.61. For the proof, we need the following lemma:

Suppose that $A_{n} \in \mathbb{E}$ for $n=1,2, \ldots$, and

$$
\sum_{n=1}^{\infty} d\left(A_{n}, A_{n+1}\right)<\infty
$$

Then the sets $\varlimsup_{n \rightarrow \infty} A_{n}$ and $\underline{\lim }_{n \rightarrow \infty} A_{n}$ are $\mu$-measurable.

Suppose first the sequence $\left\{A_{n}\right\}$ to be monotone. Then

$$
\begin{array}{cc} 
& \varlimsup_{n \rightarrow \infty} A_{n}=\varlimsup_{n \rightarrow \infty} A_{n}=\lim _{n \rightarrow \infty} A_{n}, \\
\text { and } & \lim _{n \rightarrow \infty} A_{n}=A_{1}-\left[\left(A_{1}-A_{2}\right)+\left(A_{2}-A_{3}\right)+\ldots\right] \\
\text { or } & \lim _{n \rightarrow \infty} A_{n}=A_{1}+\left(A_{2}-A_{1}\right)+\left(A_{3}-A_{2}\right)+\ldots
\end{array}
$$

The sets on the right-hand side of the above formulae being disjoint, the set $\lim _{n \rightarrow \infty} A_{n}$ is $\mu$-measurable by (15), (16) and $1.6(\mathrm{c})$. In the general case we have

$$
\left(A_{1}+A_{2}+\ldots+A_{n+1}\right)-\left(A_{1}+A_{2}+\ldots+A_{n}\right) \subset A_{n+1}-A_{n} ;
$$

thus, by what has been just proved, the set $A_{1}+A_{2}+\ldots$, and hence the sets $A_{n}+A_{n+1}+\ldots$, are $\mu$-measurable. Since

$$
\left(A_{n}+A_{n+1}+\ldots\right)-\left(A_{n+1}+A_{n+2}+\ldots\right) \subset A_{n}-A_{n+1},
$$

6) For Borel fields, in the particular case, where the set-function $\mu(E)$ is real-valued, this was proved by O. Nikodym in his paper Sur une généralisation des intégrales de M. J. Rádon, Fundamenta Mathematicae 15 1930), p. 131-179.

we see that the set $\varlimsup_{n \rightarrow \infty} A_{n}=\left(A_{1}+A_{2}+\ldots\right)\left(A_{2}+A_{3}+\ldots\right) \ldots$ is $\mu$-measurable. We prove similarly that $\varliminf_{n \rightarrow \infty} A_{n} \in \mathbb{E}$.

1.62. To prove that the function (15) satisfies the triangle-inequality, note that 1.6 (a) and 1.6(b) imply the inequality

$$
\left\|\mu\left(E_{1}+E_{2}\right)\right\| \leqslant\left\|\mu\left(E_{1}\right)\right\|+\left\|\mu\left(E_{2}\right)\right\|
$$

for any $E_{1}, E_{2} \epsilon$ E. It is sufficient to make use of the inclusion

$$
(A-C)+(C-A) \subset(A-B)+(B-A)+(B-C)+(C-B) \text {. }
$$

To prove now that the space $X(\xi)$ is complete, it will be convenient to apply the characteristic functions of sets belonging to $\mathfrak{E}$. If $h(t)$ and $g(t)$ are the characteristic functions of the sets $A$ and $B$ respectively, then $|h(t)-g(t)|$ is the characteristic function of their symmetric difference, i.e. of the set $(A-B)+(B-A)$.

Suppose that $E_{n} \in X(\mathcal{E})$ and $d\left(E_{n}, E_{m}\right) \rightarrow 0$ as $m, n \rightarrow \infty$. Let $\left\{k_{n}\right\}$ be an increasing sequence of indices such that

$$
d\left(E_{p}, E_{q}\right)<1 / 2^{n} \quad \text { for } p, q \geqslant k_{n} ;
$$

put $S_{n}=E_{k_{n}}, A_{n}=\left(S_{n}-S_{n+1}\right)+\left(S_{n+1}-S_{n}\right)$, and denote by $h_{n}(t)$ the characteristic function of the set $S_{n}$. By lemma 1.61 the sets $\underline{S}=\underline{\lim } S_{n}$ and $\bar{S}=\overline{\lim } S_{n}$ are $\mu$-measurable. Denote by $h(t)$ the

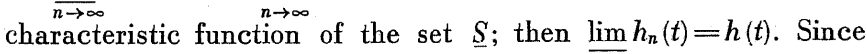

$$
m>n \text { implies }\left|h_{n}(t)-h_{m}(t)\right| \leqslant \sum_{i=n}^{m-1}\left|h_{i}(t)-h_{i+1}(t)\right|,
$$

we get

$$
\left|h_{n}(t)-h(t)\right| \leqslant \sum_{i=n}^{\infty}\left|h_{i}(t)-h_{i+1}(t)\right|
$$

hence

$$
\left(\underline{S}-S_{n}\right)+\left(S_{n}-\underline{S}\right) \subset A_{n}+A_{n+1}+\ldots,
$$

and since the sets on the right-hand side of the formula

$$
A_{n}+A_{n+1}+\ldots=A_{n}+\left(A_{n+1}-A_{n}\right)+\ldots
$$

are $\mu$-disjoint, the set $A_{n}+A_{n+1}+\ldots$ is measurable by (17) and $1.6(\mathrm{c})$, and we get

$$
\left\|\mu\left(A_{n}+A_{n+1}+\ldots\right)\right\| \leqslant\left\|\mu\left(A_{n}\right)\right\|+\sum_{k=n}^{\infty}\left\|\mu\left(A_{k+1}-A_{k}\right)\right\|<\frac{1}{2^{n-1}} .
$$


Hence

$$
d\left(S_{n}, \underline{S}\right) \leqslant \frac{1}{2^{n-1}} .
$$

Let now $\varepsilon>0$ be arbitrary, and choose $m$ so that $1 / 2^{m-2}<\varepsilon$; then $d\left(S_{m}, S\right)<\varepsilon / 2$, and by (17) we have $d\left(S_{m}, E_{n}\right)<\varepsilon / 2$ for $n \geqslant k_{m}$; it follows $d\left(E_{n}, \underline{S}\right)<\varepsilon$.

1.63. Denote by $X(T)$ the space of the real-valued functions $x=x(t)$ defined in $T$ and bounded outside null sets; with the addition of elements and the multiplication by real numbers defined as usual, and with the norm $\|x\|$ of the element $x=x(t)$ as the largest lower bound of the numbers $k$ for which $|x(t)| \leqslant k$ holds outside a null set, $X(T)$ is a Banach space.

Denote by $x_{A}=x_{A}(t)$ the characteristic function of the set $A_{\epsilon} \mathbb{E}$, and by $X_{s,}(\mathbb{E})$ the class of the functions $x_{A}$ as $A$ runs over $\mathbb{E}$. If the distance of two elements $x_{A}, x_{B} \in X_{g s}(\mathbb{E})$ is defined by the formula

$$
d\left(x_{A}, x_{B}\right)=d(A, B),
$$

we easily see by 1.62 that $X_{g s}(\mathbb{E})$ is a complete metric space.

Obviously $X_{g s}(\mathbb{E})$ is the subset of the space $X(T)$.

If $d\left(x_{A_{n}}, x_{A}\right) \rightarrow 0$, we shall write $x_{A_{n}} \stackrel{l}{\rightarrow} x_{A}$, analogously as in Saks spaces.

In the space $X_{s s}(\mathbb{E})$ we introduce the conditions $\left(\Sigma_{1}^{0}\right),\left(\Sigma_{2}^{0}\right)$ and $\left(\Sigma_{2}^{\prime}\right)$ by rewriting the conditions $\left(\Sigma_{1}\right),\left(\Sigma_{2}\right)$ and $\left(\Sigma_{2}^{\prime}\right)$ with $X_{g s}(\mathbb{E})$ instead of $X_{s}$.

We shall prove that the space $X_{g s}(\mathbb{E})$ satisfies the conditions $\left(\Sigma_{1}^{0}\right),\left(\Sigma_{2}^{0}\right)$ and $\left(\Sigma_{2}^{0^{\prime}}\right)$. Hence the space $X_{g s}(\mathbb{E})$ may be considered as a generalized Saks space. The difference between the Saks spaces and the space $X_{g s}(\mathbb{E})$ lies in the reach of the distance definition: in the former space the formula (1) defining the distance has a meaning in the whole of the fundamental Banach space $X$, and in the latter space the distance is defined in $X_{g s}(\mathbb{E})$ only.

To prove that the space $X_{g s}(\mathbb{E})$ satisfies the condition $\left(\Sigma_{1}^{0}\right)$, let 0 denote the characteristic function of the null set, and let $x_{0}=x_{A_{0}}$ be the centre of the sphere $K\left(x_{0}, \varrho\right)$. If $d\left(x_{A}, 0\right)<\delta=\varrho$, $B=A_{0}+\left(A-A_{0}\right)$, and $C=A_{0}-A$, then we have $x_{B}-x_{C}=x_{A}$, $d\left(x_{B}, x_{A_{0}}\right)=\left\|\mu\left(A-A_{0}\right)\right\| \leqslant \delta=\varrho$ and $d\left(x_{C}, x_{A_{0}}\right)=\left\|u\left(A_{0} A\right)\right\| \leqslant \delta=\varrho$.
To prove that the space $X_{g s}(\mathbb{E})$ satisfies the condition $\left(\Sigma_{2}^{0}\right)$, let $d\left(x_{E_{n}}, 0\right)=\left\|\mu\left(E_{n}\right)\right\| \rightarrow 0$, and $\varepsilon_{n} \rightarrow+0$. Pick out an increasing sequence $\left\{k_{n}\right\}$ of indices such that and put

$$
A_{n}=E_{k_{n}} \text { implies }\left\|\mu\left(A_{n+1}\right)\right\|+\left\|\mu\left(A_{n+2}\right)\right\|+\ldots \leqslant \varepsilon_{k_{n}},
$$

$$
A_{i 1}=A_{i}, \quad A_{i 2}=A_{i}-A_{i} A_{i+1}, \quad A_{i 3}=A_{i 2}-A_{i} A_{i+2}, \quad \ldots
$$

The sets $A_{i s} A_{i+s}$ and $A_{i s^{\prime}} A_{i+s^{\prime}}$ are disjoint for any $s \neq s^{\prime}$, and

$$
\sum_{s=1}^{\infty}\left\|\mu\left(A_{i s} A_{i+s}\right)\right\|<\infty \text {. }
$$

By $1.6(\mathrm{c})$ the sets $B_{i}=A_{i}-\sum_{s=1}^{\infty} A_{i s} A_{i+s}$ are $\mu$-measurable, and since the sets $B_{i}$ are disjoint and $\sum_{i=1}^{\infty}\left\|\mu\left(B_{i}\right)\right\|<\infty$, we get

$$
\begin{gathered}
d\left(x_{E_{k_{i}}}, x_{B_{i}}\right) \leqslant \varepsilon_{k_{i}} \text { for } i=1,2, \ldots, \\
x_{B_{\lambda}}=\lambda_{1} x_{B_{1}}+\lambda_{2} x_{B_{2}}+\ldots \in X_{g_{s}}(\mathbb{E}),
\end{gathered}
$$

and $\lambda_{1} x_{B_{1}}+\lambda_{2} x_{B_{2}}+\ldots+\lambda_{n} x_{B_{n}} \stackrel{l}{\rightarrow} x_{B_{\lambda}}, \lambda_{i}$ being 0 's or 1 's.

The proof of $\left(\Sigma_{2}^{0^{\prime}}\right)$ is analogous.

1.7. Now some examples of separable $X_{g s}(\mathbb{E})$ spaces will be given. Since the spaces $X_{g s}(\mathbb{E})$ arise from the spaces $X(\mathbb{E})$ by passing to characteristic functions, we indicate in each case the set $T$, the class $\mathbb{E}$ and the measure $\mu(E)$. We give also examples of denumerable and dense sets in $X(\mathbb{E})$, and we discuss further the separability of these spaces.

\section{Examples of separable $X_{g s}(\mathbb{E})$-spaces.}

$\left(I^{0}\right) T$ is a finite interval $Q$ in the space $\mathscr{E}^{n}$ (the $n$-dimensional euclidean space).

$\mathbb{E}$ is the class of Lebesgue-measurable sets in $Q$.

$\mu(E)=|E|$ is the Lebesgue-measure of $E$.

$\mathfrak{E}_{0}$ is the class of sets which are sums of a finite number of intervals with rational vertices.

(III) $T$ is the interval $(-\infty,+\infty)$.

$\mathbb{E}$ is the class of Lebesgue-measurable sets $E$ of relative measure 0 , i.e. such that $\lim _{\tau \rightarrow \infty}|\langle-\tau, \tau\rangle \cdot E| / 2 \tau=0$. 
$\mu(E)$ is the function $|\langle-\tau, \tau\rangle \cdot E| / 2 \tau$ in an interval $0<a \leqslant \tau$, the values of $\mu(E)$ being considered as elements of the Banach space composed of the continuous functions $x=x(t)$ in $\langle a, \infty)$ converging to 0 as $t \rightarrow \infty$, with the norm $\|x\|=\sup _{\langle a, \infty)}|x(t)|$.

$\mathfrak{E}_{0}$ is the class of sets which are sums of a finite number of intervals with rational end-points.

(III $\left.{ }^{0}\right) T$ is the interval $(0, \infty)$.

$\mathbb{E}$ is the class of Lebesgue-measurable sets $E$ in $(0, \infty)$ such that

$$
\lim _{\tau \rightarrow \infty} \int_{E} K(\tau, t) d t=0,
$$

where $K(\tau, t)$ is a function satysfying the conditions $(6)-\left(6^{\prime \prime}\right)$ and positive for $a \leqslant \tau$ and $0 \leqslant t<\infty$.

$\mu(E)=\int_{E} K(\tau, t) d t$; this function considered for $a \leqslant \tau$ is an element of the Banach space defined in $\left(\mathrm{II}^{\circ}\right)$.

$\mathbb{E}$ is the same set as in $\left(\mathrm{II}^{\circ}\right)$.

$\left(\mathrm{IV}^{0}\right) T$ is the set of positive integers.

$\mathcal{E}$ is the class of all subsets of $T$.

$\mu(E)=\sum_{i=1}^{\infty} \frac{1}{2^{n_{i}}}$, where $E$ is the set $\left(n_{1}, n_{2}, \ldots\right)$; thus the values of $\mu(E)$ are real numbers.

$\mathfrak{F}_{0}$ is the class of finite sets composed of positive integers.

$\left(V^{0}\right) T$ is the set of positive integers.

$\mathcal{E}$ is the class of all sets $E=\left(n_{1}, n_{2}, \ldots\right)$ of positive integers for which

$$
\lim _{i \rightarrow \infty} \sum_{n=1}^{\infty} \alpha_{i n} \lambda_{n}=0
$$

where $\alpha_{\text {in }}$ are non-negative, satisfy the conditions (3) and ( $\left.3^{\prime}\right)$, and

$$
\lambda_{n}= \begin{cases}1 & \text { for } n=n_{i}, \quad i=1,2, \ldots \\ 0 & \text { elsewhere. }\end{cases}
$$

$\mu(E)$ is the sequence $\left\{t_{i}\right\}$, where $t_{i}=\sum_{n=1}^{\infty} \alpha_{i n} \lambda_{n}$; the values of $\mu(E)$ belong to the space $\boldsymbol{m}_{0}$.

$\mathbb{E}_{0}$ is the same set as in $\left(\mathrm{IV}^{\circ}\right)$.

$\left(\mathrm{VI}^{\circ}\right) T$ is the set of positive integers.

$\mathcal{E}$ is the class of all sets $E=\left(n_{1}, n_{2}, \ldots\right)$ of positive integers for which

$$
\lim _{t \rightarrow t_{1}} \sum_{n=1}^{\infty} a_{n}(t) \lambda_{n}=0
$$

where the $a_{n}(t)$ are continuous functions, non-negative for $t \epsilon\left\langle t_{0}, t_{1}\right\rangle$ with $t_{1} \leqslant \infty$, and satisfying the conditions:

(i) $\quad \sum_{n=1}^{\infty} \alpha_{n}(t) \leqslant K \quad$ for $t \epsilon\left\langle t_{0}, t_{1}\right\rangle$,

(ii) $\lim _{t \rightarrow t_{1}} \alpha_{n}(t)=0 \quad$ for $n=1,2, \ldots$,

(iii) the series $\sum_{n=1}^{\infty} a_{n}(t)$ converges uniformly in every interval $\left\langle t_{0}, t\right) \subset\left\langle t_{0}, t_{1}\right\rangle$.

$\mu(E)=\sum_{n=1}^{\infty} \alpha_{n}(t) \lambda_{n}$, this function (continuous in $\left\langle t_{0}, t_{1}\right\rangle$ ) being considered as an element of the space $\boldsymbol{C}$.

$\mathfrak{E}_{0}$ is the same set as in $\left(\mathrm{IV}^{0}\right)$.

1.71. We presently prove that the sets $\tilde{E}_{0}$ defined above are dense in $X(\mathbb{F})$. The cases of the spaces $\left(\mathrm{II}^{\circ}\right)$, (III $\left.{ }^{\circ}\right)$ and $\left(\mathrm{VI}^{\circ}\right)$ require only some explanations.

In the case of the space $\left(\mathrm{III}^{0}\right)$ set for $n=1,2, \ldots$ and $E_{n}=E\langle 0, n\rangle$

$$
\varphi(\tau)=\int_{E_{n}} K(\tau, t) d t, \quad \varphi(\tau)=\int_{E} K(\tau, t) d t .
$$

Since the functions $\varphi_{n}(t)$ and $\varphi(t)$ are continuous and

$$
\varphi_{n}(\tau) \leqslant \varphi_{n+1}(\tau) \leqslant \varphi(\tau), \quad \varphi_{n}(\tau) \rightarrow \varphi(\tau),
$$

the sequence $\left\{\varphi_{n}(\tau)\right\}$ converges to $\varphi(t)$ uniformly in every finite interval. Moreover, since

we get

$$
\left|\varphi_{n}(\tau)-\varphi(\tau)\right| \leqslant \varphi(\tau)<\varepsilon \quad \text { for } \tau \geqslant \tau(\varepsilon),
$$

$$
\max _{\langle a, \infty\rangle}\left|\varphi_{n}(\tau)-\varphi(\tau)\right| \rightarrow 0 \text { as } n \rightarrow \infty \text {. }
$$

Choose $m$ so that

$$
d\left(E_{m}, E\right)=\max _{\langle a, \infty\rangle}\left|\varphi_{m}(\tau)-\varphi(\tau)\right|<\varepsilon / 2
$$

and let $A$ be a subset of $\langle 0, m\rangle$ composed of a finite number of intervals with rational ends such that

$$
d\left(E_{m}, A\right)=\max _{\langle a, \infty\rangle}\left|\int_{\mathcal{M}} K(\tau, t) d t\right|<\varepsilon / 2,
$$


where $M=\left(E_{m}-A\right)+\left(A-E_{m}\right)$. This is possible by Lemma $(*)$ p. 251. Hence $d(E, A) \leqslant d\left(E, E_{m}\right)+d\left(E_{m}, A\right)<\varepsilon$, and this shows that the set $\mathfrak{E}_{0}$ is dense in the set $\mathfrak{E}$.

The space $\left(\mathrm{II}^{0}\right)$ is a particular case of the space $\left(\mathrm{III}^{0}\right)$.

We pass now to the space $\left(\mathrm{VI}^{0}\right)$. Let the set $E=\left(n_{1}, n_{2}, \ldots\right)$ belong to it, and let

$\lambda_{n}=\left\{\begin{array}{l}1 \text { for } n=n_{i}, \text { where } i=1,2, \ldots \\ 0 \text { elsewhere, }\end{array} \quad \lambda_{n}^{(m)}=\left\{\begin{array}{l}\lambda_{n} \text { for } n=1,2, \ldots, m \\ 0 \text { for } n>m .\end{array}\right.\right.$

Given any $\varepsilon>0$, there exists a $t^{\prime} \epsilon\left(t_{0}, t_{1}\right)$ such that

$$
t \geqslant t^{\prime} \text { implies } \sum_{n=1}^{\infty} \alpha_{n}(t) \lambda_{n}<\varepsilon .
$$

The series $\sum_{n=1}^{\infty} a_{n}(t)$ converges uniformly in $\left\langle t_{0}, t^{\prime}\right\rangle$; hence we have $\sum_{n=1}^{\infty} \alpha_{n}(t)\left(\lambda_{n}-\lambda_{n}^{(m)}\right)<\varepsilon$ for $t \epsilon\left\langle t_{0}, t^{\prime}\right\rangle$ and $m$ sufficiently large; this implies that

$$
\max _{\left\langle t_{0}, t_{1}\right\rangle} \sum_{n=1}^{\infty} a_{n}(t)\left(\lambda_{n}-\lambda_{n}^{(m)}\right) \rightarrow 0 \text { as } m \rightarrow \infty .
$$

Thus, denoting by $E_{m}$ the set $\left(n_{1}, n_{2}, \ldots, n_{m}, 0,0, \ldots\right)$, we get $d\left(E, E_{m}\right) \rightarrow 0$, i.e. $\mathfrak{E}_{0}$ is dense in $\mathfrak{E}$.

2.1. An operation $U(x)$ from a Saks space $X_{s}$ to a Banach or Fréchet space $Y$ will be said to be additioe, if for arbitrary $x_{1}, x_{2} \in X_{s}$ and arbitrary rational $\lambda_{1}, \lambda_{2}$

$\lambda_{1} x_{1}+\lambda_{2} x_{2} \in X_{s}$ implies $U\left(\lambda_{1} x_{1}+\lambda_{2} x_{2}\right)=\lambda_{1} U\left(x_{1}\right)+\lambda_{2} U\left(x_{2}\right)$.

The operation $U(x)$ will be termed $\left(X_{s}, Y\right)$-continuous, if

$$
x_{n} \stackrel{l}{\rightarrow} x \text { implies } U\left(x_{n}\right) \rightarrow U(x)
$$

according to the convergence defined in $Y$ by the norm.

Any additive and $\left(X_{s}, Y\right)$-continuous operation will be said to be $\left(X_{s}, Y\right)$-linear.

If $U(x)$ is a functional, and when no ambiguity arises, we shall simply say continuous operation or linear operation instead of $\left(X_{s}, Y\right)$-continuous operation or $\left(X_{s}, Y\right)$-linear operation respectively.

If $U(x)$ is an operation defined in a Banach or Fréchet space, a corresponding terminology will be used.
2.11. A set-operation $U(E)$ from a space $X(\mathcal{E})$ to a Banach or Fréchet space will be said to be continuous in $X(\mathbb{E})$, if

$$
\mu\left(E_{n}\right) \rightarrow 0 \text { implies } U\left(E_{n}\right) \rightarrow 0 .
$$

It will be called additive if

$$
U\left(E_{1}+E_{2}\right)=U\left(E_{1}\right)+U\left(E_{2}\right),
$$

$E_{1}$ and $E_{2}$ being any $\mu$-disjoint sets; this condition implies that $U(E)=0$ for any null set $E$.

If $U\left(E_{1}+E_{2}+\ldots\right)=U\left(E_{1}\right)+U\left(E_{2}\right)+\ldots$ for every sequence of mutually $\mu$-disjoint sets of $X(\mathbb{E})$ the sum of which belongs to $X(\widetilde{E})$, the operation $U(E)$ will be termed completely additive.

An analogous terminology will be used in the isomorphical space $X_{g s}(\mathbb{E})$ of the characteristic functions.

2.2. We now prove two lemmas:

(A) Let $U_{n}(x)$ be additive operations from $X_{s}$ to a Fréchet space. Each of the folloming conditions is sufficient for the sequence $U_{n}(x)$ to be equicontinuous at any point $x \in X_{\mathbf{s}}$ :

(a') the space $X_{s}$ satisfies the condition $\left(\Sigma_{1}\right)$, and there exists an element $x_{0}$ at rohich the operations $U_{n}(x)$ are equicontinuous, $\left(\mathrm{a}^{\prime \prime}\right)$ the space $X_{s}$ is arbitrary, and there exists a $x_{0}$ such that $\left\|x_{0}\right\|<1$ and that the operations $U_{n}(x)$ are equicontinuous at $x_{0}$.

Suppose the condition $\left(\mathrm{a}^{\prime}\right)$ is satisfied. Given any $\varepsilon>0$ there exists a $\varrho(\varepsilon)>0$ such that

$$
x \in K\left(x_{0}, \varrho(\varepsilon)\right) \text { implies }\left\|U_{n}(x)-U_{n}\left(x_{0}\right)\right\|<\varepsilon / 2 \text { for } n=1,2, \ldots
$$

By $\left(\Sigma_{1}\right)$ there exists a $\delta(\varepsilon)>0$ such that any element $y$, for which $d(y, 0)<\delta(\varepsilon)$, is of the form $y=x_{1}-x_{2}$ with $x_{1}, x_{2} \in K\left(x_{0}, \varrho(\varepsilon)\right)$. The operation $U_{n}(x)$ being additive, we have

$$
\left\|U_{n}(y)\right\| \leqslant\left\|U_{n}\left(x_{1}\right)-U_{n}\left(x_{2}\right)\right\|<\varepsilon .
$$

Let $\hat{x}$ be an arbitrary element of $X_{s}$; by 1.22 there exists a $\delta_{1}(\varepsilon)>0$ such that

$$
\|x-\hat{x}\|^{*}<\delta_{1}(\varepsilon) \text { implies }\left\|\frac{x-\hat{x}}{2}\right\|^{*}<\delta(\varepsilon) .
$$

Since $(x-\hat{x}) / 2 \in X_{s}$, we get for $n=1,2, \ldots$

$$
\left\|\frac{1}{2} U_{n}(x)-\frac{1}{2} U_{n}(\hat{x})\right\|=\left\|U_{n}\left(\frac{x-\hat{x}}{2}\right)\right\|<\varepsilon, \quad\left\|U_{n}(x)-U_{n}(\hat{x})\right\|<2 \varepsilon .
$$


Suppose now the condition (a") to be satisfied. Let $X_{s}$ be arbitrary, $\eta$ any positive, and $\lambda$ a rational number satisfying the inequality $\left\|x_{0}\right\|<\lambda<1$. Then there exists an $\varepsilon>0$ such that

$$
\|z\|<\varepsilon \text { and } z \in Y \text { imply }\left\|\frac{1}{1-\lambda} z\right\|<\eta \text {. }
$$

If $y \in X_{s}$, then $x_{0}+(1-\lambda) y \in X_{s}$ and $d(y, 0)<\delta(\varepsilon)$ imply

$$
x_{0}+(1-\lambda) y \in K\left(x_{0}, \delta(\varepsilon)\right) \text {. }
$$

Here we can take for $K\left(x_{0}, \varrho(\varepsilon)\right)$ the same sphere as in the proof of the first part of the lemma; then for $n=1,2, \ldots$

$\left\|U_{n}\left(x_{0}+(1-\lambda) y\right)-U_{n}\left(x_{0}\right)\right\|=\left\|(1-\lambda) U_{n}(y)\right\|<\varepsilon, \quad\left\|U_{n}(y)\right\|<\eta$.

Hence the operations are equicontinuous at 0 . We complete the proof as above.

In connection with lemma (A) note that the set $X^{*}$ of those elements, for which $\|x\|<1$, is a $F_{\sigma}$ in $X_{s}$. Remark that

$$
x_{n} \rightarrow x_{0} \text { implies } \underset{n \rightarrow \infty}{\lim }\left\|x_{n}\right\| \geqslant\left\|x_{0}\right\| \text {. }
$$

In fact, suppose $\left\|x_{0}\right\|>0$, and choose $\varepsilon>0$ so as $0<\varepsilon<\left\|x_{0}\right\|$ and $\left\|x_{n_{i}}\right\|<\left\|x_{0}\right\|-\varepsilon=\eta$; since $\left\|x_{n_{i}} / \eta\right\|<1$, we get $x_{n_{i}} / \eta \stackrel{l}{\rightarrow} y$ and $y \in X_{s}$; on the other hand we have obviously $y=x_{0} / \eta$, and this leads to a contradiction: $\left\|x_{0}\right\| \leqslant \eta$. The sets $X_{n}$ of the elements $x$ satisfying the inequality $\|x\| \leqslant 1-1 / n$ being closed in $X_{s}$, we see that $X^{*}$ is a $F_{\sigma}$.

Applying this result it can be easily proved that in the spaces (I)-(VII) defined in 1.4 the set $X^{*}$ is of the first category of Baire.

(B) Let $\left(a_{i n}\right)$ be a matrix of real numbers such that $\sum_{n=1}^{\infty}\left|a_{i n}\right|<\infty$ for $i=1,2, \ldots \quad$ and $\quad \lim _{i \rightarrow \infty} a_{i n}=a_{n}$ for $n=1,2, \ldots$

If for any sequence $\left\{\lambda_{n}\right\}$ composed of 0 's and 1's there exists the limit $\lim _{i \rightarrow \infty} \sum_{n=1}^{\infty} \lambda_{n} a_{i n}$, then $\lim _{n \rightarrow \infty} \sup _{(i)}\left|a_{i n}\right|=0^{7}$ ).

It is sufficient to consider the case $a_{n}=0$. Suppose that $\varlimsup_{n \rightarrow \infty} \sup _{(i)}\left|a_{i n}\right|>r>0$; then we can define successively two increasing sequences of indices, $\left\{\boldsymbol{i}_{k}\right\}$ and $\left\{\boldsymbol{n}_{k}\right\}$, such that

7) This lemma is known.

$$
\begin{array}{r}
1^{0} \sum_{n=n_{k+1}}^{\infty}\left|a_{i_{k}}\right|<\frac{r}{8}, \quad 2^{0} \quad\left|a_{i_{k} n_{k}}\right|>\frac{3}{4} r, \\
\text { Put } \\
\tilde{\lambda}_{n}=\left\{\begin{array}{cc}
\frac{1}{2}\left[(-1)^{k}+1\right] & \text { for } n=n_{k} \\
0 & \text { for } n \neq n_{k} ;
\end{array}\right.
\end{array}
$$

$k$ being even, we have

$$
\sum_{n=1}^{\infty} \tilde{\lambda}_{n} a_{i_{k} n}=\sum_{n=1}^{n_{k}-1} \tilde{\lambda}_{n} a_{i_{k} n}+\tilde{\lambda}_{n_{k}} a_{i_{k} n_{k}}+\sum_{n=n_{k+1}}^{\infty} \tilde{\lambda}_{n} a_{i_{k} n} \begin{cases}>\frac{r}{2} & \text { if } a_{i_{k} n_{k}}>0, \\ <-\frac{r}{2} \text { if } a_{i_{k} n_{k}}<0,\end{cases}
$$

and $k$ being odd, we have

$$
\left|\sum_{n=1}^{\infty} \tilde{\lambda}_{n} a_{i_{k} n}\right| \leqslant \frac{r}{8}+\frac{r}{8}=\frac{r}{4} .
$$

The sequence $\sum_{n=1}^{\infty} \tilde{\lambda}_{n} a_{i n}=b_{i}$ is divergent, and this is contradictory.

2.3. Denote by $Y$ the space conjugate to a Banach space $Y$. The subset $Y_{0}$ of $Y$ is called fundamental, if there exist two positive constants $c$ and $C$ such that

$\left(f_{1}\right) \sup |\eta(y)| \geqslant c\|y\|$ for any $y \in Y$,

$$
\left.\mathrm{f}_{2}\right)^{\eta \in Y_{0}}\|\eta\| \leqslant C \quad \text { for each } \eta \in Y_{0} \text {. }
$$

Theorem 1. If the space $X_{s}$ satisfies the condition $\left(\Sigma_{1}\right)$ or the condition $\left(\Sigma_{s}\right)$, then the folloming conditions are jointly sufficient for an additive operation to be $\left(X_{s}, Y\right)$-linear:

$\left(a_{1}\right)$ the space $Y$ is a separable Banach space,

$\left(b_{1}\right)$ there exists a fundamental set $Y_{0}$ such that for any $\eta \in Y_{0}$ the functional $\eta(U(x))$ is continuous in $X_{s}$.

Proof. Suppose first the space $X_{s}$ satisfies the condition $\left(\Sigma_{1}\right)$. By $\left(a_{1}\right),\left(b_{1}\right)$ and a general theorem ${ }^{8}$ ) the operation $U(x)$ is of By $\left(a_{1}\right),\left(b_{1}\right)$ and a general theorem in a residual set. It is sufBaire's first class, hence continuous
ficient to apply the lemma $2.2(\mathrm{~A})$.

icient to apply the lemo 8) A. Alexiewicz and W. Orlicz, Sur la continuité et la classification théorème 1 . 
Suppose now the space $X_{s}$ satisfies the condition $\left(\Sigma_{2}\right)$.

By the condition $\left(b_{1}\right)$ the convergence $x_{n} \stackrel{l}{\rightarrow} x_{0}$ implies that

$$
\varliminf_{n \rightarrow \infty}\left\|U\left(x_{n}\right)\right\| \geqslant \frac{c}{C}\left\|U\left(x_{0}\right)\right\|=k\left\|U\left(x_{0}\right)\right\| .
$$

By lemma 2.2(A) it is sufficient to prove that the operation $U(x)$ is continuous at 0 . Suppose it is not the case; then there exists an $\varepsilon_{0}>0$ and a $x_{n}$ such that $x_{n} \stackrel{l}{\rightarrow} 0$ and $\left\|U\left(x_{n}\right)\right\| \geqslant \varepsilon_{0}$. By $\left(\boldsymbol{\Sigma}_{2}\right)$ and (19) there exists a sequence $\left\{k_{n}\right\}$ of indices and a sequence $\left\{\hat{x}_{k_{n}}\right\}$ of elements such that the conditions (ii) and (iii) of $\left(\Sigma_{2}\right)$ are satisfied, and

$$
\left\|U\left(\hat{x}_{k_{n}}\right)\right\| \geqslant k \frac{\varepsilon_{0}}{2} \quad \text { for } n=1,2, \ldots
$$

Let $\eta \in Y_{0} ; \hat{x}_{2}$ being the element the existence of which is assured by the condition (iii) of $\left(\Sigma_{2}\right)$, we have by $\left(b_{1}\right)$

$$
\sum_{n=1}^{\infty} \lambda_{n} \eta\left(U\left(\hat{x}_{k_{n}}\right)\right)=\eta\left(U\left(\hat{x}_{\lambda}\right)\right) \text {. }
$$

The set $Y_{0}$ being fundamental, there exists a sequence $\left\{\eta_{i}\right\}$ of elements of $r_{0}$ such that

$$
\left|\eta_{i}\left(U\left(\hat{x}_{k_{i}}\right)\right)\right| \geqslant \frac{c}{2}\left\|U\left(\hat{x}_{k_{i}}\right)\right\| \geqslant k \frac{c \varepsilon_{0}}{4} \quad \text { for } i=1,2, \ldots
$$

and by the separability of the space $Y$ we can suppose that there exists a subsequence $\left\{l_{i}\right\}$ such that $\lim _{i \rightarrow \infty} \eta_{l_{i}}(y)$ exists for every $y \in Y$. Write $a_{i n}=\eta_{l_{i}}\left(U\left(\hat{x}_{k_{l_{n}}}\right)\right)$; the matrix $\left(a_{i n}\right)$ satisfies by (20) the hypotheses of lemma $2.2(\mathrm{~B})$; hence $\left|\eta_{l_{i}}\left(U\left(\hat{x}_{k_{l_{i}}}\right)\right)\right| \rightarrow 0$, which is impossible, since $\mid \eta_{t_{i}}\left(U\left(\hat{x}_{k_{l_{i}}}\right) \mid \geqslant k \frac{c \varepsilon_{0}}{4}\right.$.

Theorem 1'. If the space $X_{s}$ is separable and satisfies the condition $\left(\Sigma_{1}\right)$ or the condition $\left(\Sigma_{2}\right)$, the folloming conditions are jointly sufficient for an additive operation $U(x)$ to be $\left(X_{s}, Y\right)$-linear:

$\left(\mathrm{a}_{1}^{\prime}\right) \boldsymbol{Y}$ is a Banach space,

$\left(b_{1}^{\prime}\right)$ for any linear functional $\eta(y)$ the functional $\eta(U(x))$ is linear in $\bar{X}_{\mathrm{s}}$.
Proof. Let the sequence $\left\{x_{n}\right\}$ be dense in $X_{s} ; x$ being any element of $X_{s}$, choose $x_{n_{i}} \stackrel{l}{\rightarrow} x$. Since by $\left(b_{i}^{\prime}\right) \eta\left(U\left(x_{n_{i}}\right)\right) \rightarrow \eta(U(x))$ for every linear functional $\eta$, there exists by a well-known theorem a sequence of linear combinations of the elements $U\left(x_{n_{i}}\right)$ converging to $U(x)$ in $Y$. This implies that the range of operation $U(x)$ belongs to a separable subspace $Y_{0} \subset Y$, and it suffices to apply Theorem 1.

Theorem 2. If the space $X_{s}$ satisfies the condition $\left(\Sigma_{1}\right)$ or the condition $\left(\Sigma_{2}\right)$, and $U(x)$ is an additive operation in $X_{s}$, then the folloming conditions are jointly sufficient for $U(x)$ to transform any set $X_{0}$ bounded rith respect to the norm \|\|$^{*}$ into a bounded set:

$\left(\mathrm{a}_{2}\right) Y$ is a Fréchet space,

$\left(\mathrm{b}_{2}\right) x_{n} \stackrel{l}{\rightarrow} x_{0}$ implies

$$
\varliminf_{n \rightarrow \infty}\left\|U\left(x_{n}\right)\right\| \geqslant\left\|U\left(x_{0}\right)\right\| .
$$

Proof. Suppose first the space $X_{s}$ satisfies the condition $\left(\Sigma_{1}\right)$; $\varepsilon$ being any positive number, denote by $X_{n}$ the set of the elements of $X_{s}$ such that

$$
|\vartheta| \leqslant 1 / n \text { implies }\|\vartheta U(x)\| \leqslant \varepsilon / 2 .
$$

From the additiveness of $U(x)$ and from $\left(b_{2}\right)$ it follows that for every rational $\vartheta$

$$
x_{n} \stackrel{l}{\rightarrow} x \text { and }|\vartheta| \leqslant 1 \quad \text { imply } \underset{n \rightarrow \infty}{\varliminf_{n \rightarrow \infty}}\left\|\vartheta U\left(x_{n}\right)\right\| \geqslant\|\vartheta U(x)\| .
$$

Thus the sets $X_{s}$ are closed; since $X_{s}=\sum_{n=1}^{\infty} X_{n}$, one of the sets, say $X_{p}$, contains the sphere $K\left(x_{0}, \varrho\right)$. By condition $\left(\Sigma_{1}\right)$ there exists a $\delta>0$ such that $\|y\|^{*}<\delta$, and $y \in X_{s}$ implies the existence of $x_{1}, x_{2} \in K\left(x_{0}, \varrho\right)$ for which $y=x_{1}-x_{2}$. Hence

$$
\|\vartheta U(y)\| \leqslant\left\|\vartheta U\left(x_{1}\right)\right\|+\left\|\vartheta U\left(x_{2}\right)\right\| \leqslant \varepsilon \quad \text { for }|\vartheta| \leqslant 1 / p .
$$

There exists a rational number $\vartheta_{0}$ such that $0<\vartheta_{0}<1$ and $\left\|\vartheta_{0} x\right\|^{*}<\delta$ for every $x \in X_{0}$. The inequality $|\vartheta| \leqslant \vartheta_{0} / p$ implies for every $x \in X_{0}$

$$
\|\vartheta U(x)\|=\left\|\frac{\vartheta}{\vartheta_{0}} U\left(\vartheta_{0} x\right)\right\| \leqslant \varepsilon .
$$

Thus the operation $U(x)$ maps the set $X_{0}$ into a bounded set. Suppose now the space $X_{s}$ satisfies the condition $\left(\Sigma_{2}\right)$. We first prove that $x_{n} \rightarrow 0$ implies the boundedness of the sequence $\left\{U\left(x_{n}\right)\right\}$. 
Suppose it is not the case for a sequence $\left\{x_{n}\right\} l$-converging to 0 ; then there exists an $\varepsilon_{0}>0$ and rational numbers $\vartheta_{n} \rightarrow 0$ such that $\left|\vartheta_{n}\right|<1$ and $\varlimsup_{n \rightarrow \infty}\left\|\vartheta_{n} U\left(x_{n}\right)\right\|>\varepsilon_{0}$.

By $\left(\Sigma_{2}\right),\left(b_{2}\right)$ and 1.22 there exists a sequence $\left\{k_{n}\right\}$ of indices and a sequence $\left\{\hat{x}_{k_{n}}\right\}$ of elements such that the conditions (i)-(iii) of $\left(\Sigma_{2}\right)$ are satisfied, and that

$1^{0}\left\|\vartheta_{k_{n}} U\left(\hat{x}_{k_{n}}\right)\right\| \geqslant \varepsilon_{0} / 2$ for $n=1,2, \ldots$,

$2^{0}$ the series $\sum_{n=1}^{\infty}\left\|\tau_{n} \hat{x}_{k_{n}}\right\|^{*}$ converges uniformly in the set of sequences $\left\{\tau_{n}\right\}$ with $\left|\tau_{n}\right| \leqslant 1$.

The condition (ii) of $\left(\Sigma_{2}\right)$ implies that, given any functional $\xi(x)$ of norm 1 linear in $X$,

$$
\lambda_{n}=0 \text { or } 1 \text { implies } \sum_{n=1}^{\infty} \lambda_{n}\left|\xi\left(\hat{x}_{k_{n}}\right)\right| \leqslant 1 \text {; }
$$

hence for any sequence $a=\left\{a_{n}\right\}$ of numbers absolutely less than 1 we have

$$
\left\|\sum_{n=1}^{i} a_{n} \hat{x}_{k_{n}}\right\| \leqslant 1 \text {. }
$$

Thus, by the completeness of $X_{s}$ and by $2^{\circ}$ there exists a $x_{a} \in X_{s}$ such that

$$
\sum_{n=1}^{i} a_{n} \hat{x}_{k_{n}} \stackrel{l}{\rightarrow} x_{a}
$$

We now define in the space $(\mathrm{I})$ an operation $V(a)$ writing

$$
V(a)=U\left(x_{a}\right) \quad \text { for } a=\left\{a_{n}\right\} .
$$

It is an additive operation, and by $2^{\circ}$ and $\left(b_{2}\right)$ we easily infer that, if $a_{n} \stackrel{l}{\rightarrow} a$ in the space (I), then $\lim _{n \rightarrow \infty}\left\|V\left(a_{n}\right)\right\| \geqslant\|V(a)\|$. This space satisfying the condition $\left(\Sigma_{1}\right)$ and being bounded with respect to the norm \|\|$^{*}$, the range of the operation $V(a)=U\left(x_{a}\right)$ is bounded. Hence the sequence $\left\{U\left(\hat{x}_{k_{n}}\right)\right\}$ is bounded. But this contradicts $1^{0}$.

Let now $x_{n} \in X_{0}, \vartheta_{n} \rightarrow 0$, and let $\vartheta_{n}^{\prime}$ be rational numbers such that $\vartheta_{n}^{\prime} \rightarrow 0$ and $1>\left|\vartheta_{n} / \vartheta_{n}^{\prime}\right| \rightarrow 0$. Since $\vartheta_{n}^{\prime} x_{n} \rightarrow 0$, we get

which completes the proof.
In connection with Theorem 2 we prove the following one:

If the operation $U(x)$ is additive, and maps any bounded set into a bounded set, then

$$
x \in X_{s} \text { and } \vartheta x \in X_{s} \text { imply } U(\vartheta x)=\vartheta U(x) .
$$

It is sufficient to prove that $\vartheta_{n} \rightarrow 0$ implies $U\left(\vartheta_{n} x\right) \rightarrow 0$.

Let $\vartheta_{n}^{\prime}$ be rational numbers such that $\left|\vartheta_{n} / \vartheta_{n}^{\prime}\right| \rightarrow 0$ and $\vartheta_{n}^{\prime} \rightarrow 0$. The sequence $\left\{\frac{\vartheta_{n}}{\vartheta_{n}^{\prime}} x\right\}$ being bounded, we get $U\left(\vartheta_{n} x\right)=\vartheta_{n}^{\prime} U\left(\frac{\vartheta_{n}}{\vartheta_{n}^{\prime}} x\right) \rightarrow 0$.

Simple examples show that there exist operations additive in Saks spaces $X_{s}$ satisfying the conditions $\left(\Sigma_{1}\right)$ and $\left(\Sigma_{2}\right)$, mapping the space $X_{s}$ into a bounded set, but discontinuous in $X_{s}$.

2.4.1. We will say that a Fréchet space $Y$ satisfies the condition (Z) if boundedness of all the sums $\sum_{n=1}^{i} \lambda_{n} y_{n}$ with $\lambda_{n}=0$ or 1 implies the convergence in $Y$ of the series $\sum_{n=1}^{\infty} y_{n}{ }^{9}$ ).

Theorem 3. If the space $X_{s}$ satisfies the condition $\left(\Sigma_{2}\right)$, then the conditions $\left(\mathrm{a}_{2}\right),\left(\mathrm{b}_{2}\right)$ of Theorem 2 and the folloming condition:

$\left(\mathrm{c}_{3}\right)$ the space $Y$ satisfies the condition $(\mathrm{Z})$ are jointly sufficient for an operation $U(x)$ additioe in $X_{s}$ to be $\left(X_{s}, Y\right)$-linear.

Y)-linear.
Proof. By Lemma 2.2(A) it is sufficient to prove that $x_{n} \stackrel{l}{\rightarrow} 0$ implies $U\left(x_{n}\right) \rightarrow 0$.

Suppose it is not the case; then there exists an $\varepsilon_{0}>0$ and a sequence $x_{n} \stackrel{l}{\rightarrow} 0$ for which $\left\|U\left(x_{n}\right)\right\| \geqslant \varepsilon_{0}$. The conditions $\left(\Sigma_{2}\right)$ and $\left(b_{2}\right)$ imply the existence of a sequence $\left\{k_{n}\right\}$ of indices and of a sequence $\left\{\hat{x}_{k_{n}}\right\}$ of elements satisfying the conditions (ii) and (iii) of $\left(\Sigma_{2}\right)$, the condition $2^{0}$, p. 270 , and the following one:

$$
\left\|U\left(\hat{x}_{k_{n}}\right)\right\| \geqslant \varepsilon_{0} / 2 \text { for } n=1,2, \ldots
$$

From these conditions it follows that the partial sums of the series $\sum_{n=1}^{\infty} \lambda_{n} \hat{x}_{k_{n}}$, where $\lambda_{n}=0$ or 1 , are bounded with respect to the

9) This condition was introduced in my paper Sur les opérations linéaires dans l'espace des fonctions bornées, Studia Mathematica 10 (1948), p. 60-89. 
norm \| $\|^{*}$; hence by Theorem 2 the sequence of values

$$
U\left(\sum_{n=1}^{i} \lambda_{n} \hat{x}_{k_{n}}\right)=\sum_{n=1}^{i} \lambda_{n} U\left(\hat{x}_{k_{n}}\right)
$$

where $\lambda_{n}=0$ or 1 , is bounded. It follows by $(Z)$ that $U\left(\hat{x}_{k_{n}}\right) \rightarrow 0$, which is contradictory.

Theorem 3'. If the space $Y$ is a Banach space, and the space $X_{s}$ satisfies the condition $\left(\Sigma_{2}\right)$, then the conditions $\left(b_{1}\right)$ and $\left(c_{3}\right)$ suffice jointly for an additive operation $U(x)$ to be $\left(X_{s}, Y\right)$-linear.

Proof. Write

$$
\sup _{\eta \in Y_{0}}|\eta(y)|=\|y\|_{0}
$$

The norms || $\|$ and \|\|$_{0}$ are equivalent by virtue of conditions $\left(f_{1}\right)$ and $\left(f_{2}\right)$, p. 267. We prove that

$$
x_{n} \stackrel{l}{\rightarrow} x \text { implies }\|U(x)\|_{0} \leqslant \underline{\lim }_{n \rightarrow \infty}\left\|U\left(x_{n}\right)\right\|_{0} .
$$

In the contrary case we must have for a sequence $\left\{x_{n_{i}}\right\}$

$$
\left\|U\left(x_{n_{i}}\right)\right\|_{0} \leqslant\|U(x)\|_{0}-\varepsilon .
$$

But $\left(\mathrm{b}_{1}\right)$ implies $\eta\left(U\left(x_{n_{i}}\right)\right) \rightarrow \eta(U(x))$ for every $\eta \epsilon \Upsilon_{0}$; hence choosing $\eta \epsilon \Upsilon_{0}$ so that

we get

$$
|\eta(U(x))| \geqslant\|U(x)\|_{0}-\varepsilon / 2
$$

$$
\|U(x)\|_{0}-\varepsilon \geqslant\left\|U\left(x_{n_{i}}\right)\right\|_{0} \geqslant\left|\eta\left(U\left(x_{n_{i}}\right)\right)\right| \rightarrow|\eta(U(x))|,
$$

which is impossible. We complete the proof by applying Theorem 3.

(Reçu par la Rédaction le 20. 3. 1950). 